\title{
Early Life Nociception is Influenced by Peripheral Growth Hormone Signaling
}

\author{
Adam J. Dourson, ${ }^{1}$ Zachary K. Ford, ${ }^{1}$ Kathryn J. Green, ${ }^{1}$ Carolyn E. McCrossan, ${ }^{1}$ Megan C. Hofmann, ${ }^{1}$ \\ Renita C. Hudgins, ${ }^{1}$ and ${ }^{\circledR}$ Michael P. Jankowski ${ }^{1,2}$ \\ ${ }^{1}$ Department of Anesthesia, Division of Pain Management, Cincinnati Children's Hospital Medical Center, Cincinnati, Ohio 45229, and ${ }^{2}$ Department \\ of Pediatrics, University of Cincinnati College of Medicine, Cincinnati, Ohio 45229
}

\begin{abstract}
A number of cellular systems work in concert to modulate nociceptive processing in the periphery, but the mechanisms that regulate neonatal nociception may be distinct compared with adults. Our previous work indicated a relationship between neonatal hypersensitivity and growth hormone $(\mathrm{GH})$ signaling. Here, we explored the peripheral mechanisms by which GH modulated neonatal nociception under normal and injury conditions (incision) in male and female mice. We found that GH receptor (GHr) signaling in primary afferents maintains a tonic inhibition of peripheral hypersensitivity. After injury, a macrophage dependent displacement of injury-site GH was found to modulate neuronal transcription at least in part via serum response factor (SRF) regulation. A single GH injection into the injured hindpaw muscle effectively restored available GH signaling to neurons and prevented acute pain-like behaviors, primary afferent sensitization, and neuronal gene expression changes. GH treatment also inhibited long-term somatosensory changes observed after repeated peripheral insult. Results may indicate a novel mechanism of neonatal nociception.
\end{abstract}

Key words: dorsal root ganglion electrophysiology; hormones; macrophage; neonatal; nociception

\section{Significance Statement}

Although it is noted that mechanisms of pain development in early life are unique compared with adults, little research focuses on neonatal-specific peripheral mechanisms of nociception. This gap is evident in the lack of specialized care for infants following an injury including surgeries. This report evaluates how distinct cellular systems in the periphery including the endocrine, immune and nervous systems work together to modulate neonatal-specific nociception. We uncovered a novel mechanism by which muscle injury induces a macrophage-dependent sequestration of peripheral growth hormone (GH) that effectively removes its normal tonic inhibition of neonatal nociceptors to promote acute pain-like behaviors. Results indicate a possible new strategy for treatment of neonatal postsurgical pain.

\section{Introduction}

Functional restoration after injury requires a coordinated response between immune cells, neurons, and local tissues within the affected area. This response further generates a nociceptive signal via primary sensory neurons that is required to inform the organism of the ongoing repair process (Basbaum et al., 2009; Baoge et al., 2012; Philippou et al., 2012; Sass et al., 2018). Responses from each of these individual cell types play a role in

Received Dec. 7, 2020; revised Mar. 8, 2021; accepted Mar. 9, 2021.

Author contributions: A.J.D. and M.P.J. designed research; A.J.D., Z.K.F., K.J.G., C.E.M., M.C.H., R.C.H., and M.P.J. performed research; A.J.D. and M.P.J. analyzed data; A.J.D. and M.P.J. wrote the paper.

This work was supported by National Institutes of Health Grants R01NS105715 and R56NS103178 (to M.P.J.) and by the Rita Allen Foundation in addition to support from the Cincinnati Children's Research Foundation and Department of Anesthesia. We thank Dr. Ram Menon from the University of Michigan for supplying us with the macrophage-specific GH receptor knock-out mice.

The authors declare no competing financial interests.

Correspondence should be addressed to Michael P. Jankowski at michael.jankowski@cchmc.org.

https://doi.org/10.1523/JNEUROSCI.3081-20.2021

Copyright $\odot 2021$ the authors how noxious signals are transduced into the CNS (Basbaum et al., 2009).

The immature dorsal root ganglion (DRG) contains a compilation of sensory neuron subtypes that is distinct from adults (Jankowski et al., 2014; Sharma et al., 2020). As such, neonates are particularly vulnerable to sensory impairment during developmental injury (Lim and Godambe, 2017). Recent work in animals (Ren et al., 2004; Walker et al., 2009) and humans (Hermann et al., 2006; Walker et al., 2018; Walker, 2019) indicates that early life injury enhances pain-related responses later in life. These "priming" effects have been linked to alterations in the central nervous system (Baccei, 2016; Walker et al., 2016; Brewer and Baccei, 2020), but the peripheral component is less studied (Moriarty et al., 2018). We have shown that the pattern of primary afferent sensitization after neonatal injury is unique to that observed in adult nociceptors (Koerber and Woodbury, 2002; Ye and Woodbury, 2010; Koerber et al., 2010; Jankowski et al., 2014). Other data suggest that there is hyperinnervation of the injured tissue after an early life injury (De Lima et al., 1999; 
Brewer et al., 2020). Macrophages, in particular, also release both pro-inflammatory and anti-inflammatory signals on acute stimulation in neonates, as opposed to the sequential response in mature cells (McGrath-Morrow et al., 2015; McKelvey et al., 2015; Kumar and Bhat, 2016). This suggests that the mechanisms of nociception in neonates may be somewhat distinct.

We recently found that growth hormone (GH) may be one factor involved in generalized pain-related responses to injury in neonates (Liu et al., 2017; Ford et al., 2019). The largest increases in systemic GH levels occur during early postnatal development, which corresponds with the most rapid growth period (Bartholomew and Nath, 2009). This is the same developmental period when GH was found to influence pain-like responses and primary afferent function (Liu et al., 2017; Ford et al., 2019). GH receptors (GHrs) typically affect cellular functions through activation of various transcription factors like the signal transducers and activators of transcription (STATs), serum response factor (SRF) or ERK-like kinases (ELKs; Ceseña et al., 2007). These factors can also be invoked in response to an aversive stimulus and can modulate both transcriptional and behavioral phenotypes (Gomez et al., 2018; Salaffi et al., 2018). Immune cells that infiltrate injury sites release cytokines and growth factors during repair that can themselves be pro-nociceptive (McMahon et al., 2005, 2015; Basbaum et al., 2009; Ren and Dubner, 2010; Philippou et al., 2012). Macrophages in particular are known to use peripheral GH to modulate local inflammation (Strous et al., 1996; Govers et al., 1999; Lu et al., 2013; Schneider et al., 2019). Together, altered GH signaling within primary afferent neurons may modulate peripheral sensitization in coordination with the immune system.

Clinical reports show that in addition to growth problems, many children with GH deficiency (Dattani and Preece, 2004; Cuatrecasas, 2009; Pinho-Ribeiro et al., 2018) report pain (Cimaz et al., 2001). Other studies have found that exogenous $\mathrm{GH}$ treatment may be an effective pain therapy for patients with erythromelalgia (Cimaz et al., 2001), fibromyalgia (Cuatrecasas et al., 2007, 2010, 2012, 2014) or low back pain (Dubick et al., 2015). Conversely, GHr blockers, used to treat acromegaly, can produce pain (van der Lely et al., 2001). Thus, understanding the mechanisms of how GH regulates pain-like responses may help us understand basic nociceptive processing in the immature nervous system.

\section{Materials and Methods \\ Animals}

Male and female mice that were postnatal day (P)7-P14 ( $\pm 1 \mathrm{~d}$ around the specified age range) or P35-P56 were used throughout all experiments. In all cases except for experiments regarding SRF knock-down, no sex differences were observed and thus data are combined from both males and females for ease of presentation. Neonatal animals were kept with the dam and only separated for short durations at a time $(<1.5 \mathrm{~h})$ to perform behavioral experiments. All mice were kept in an environment-controlled facility at Cincinnati Children's Hospital Medical Center (CCHMC) with free access to food and water while on a 12/12 h light/dark cycle. Animals are defined as wild-type (WT) controls, heterozygous $(+/-)$, or homozygous $(-/-)$ for the genetic manipulation throughout all experiments.

Swiss Webster mice were born in house or purchased from Charles River or Envigo and were used as an outbred strain for most experimentation. Littermate and non-littermate C57BL/6 animals born in-house were used as controls for genetic lines bred on that background. A deletion of the GHr specifically on macrophages was kindly gifted to use by Ram Menon (University of Michigan). This mouse was generated by crossing a LysM-Cre positive animal (see Jax stock \#004781) with a GHr floxed animal to induce cell type-specific genetic deletion of $\mathrm{GHr}$ in monocytes/macrophages. Animals with a knock out of the GH-releasing hormone receptor (GHRHr) were purchased from The Jackson

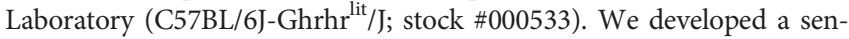
sory neuron-specific deletion of $\mathrm{GHr}$ by crossing a tamoxifen inducible Cre recombinase driven by the Advillin (Adv) promotor purchased from The Jackson Laboratory (Advillin-CreERT2; stock \#026516). Mice were crossed in-house with cryo-recovered (CCHMC Transgenic Core Facility) GHr floxed (GHr ${ }^{\text {tmlb(KOMP)Wtsi }}$ ) embryos purchased from the KOMP Repository (design ID \#49728) to make an inducible sensory neuron-specific GHr knock-out $\left(\mathrm{Adv} ; \mathrm{GHr}^{\mathrm{f} / \mathrm{f}}\right)$. A Cre-dependent reporter mouse was also used to drive tdTomato (tdTOM) expression (B6.Cg-Gt

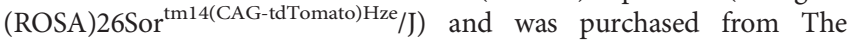
Jackson Laboratory (stock \#007914). Finally, LysM-Cre animals purchased from The Jackson Laboratory (stock \#004781) were crossed to the tdTom mice to generate myeloid/macrophage reporter mice. All procedures were approved by the CCHMC Institutional Animal Care and Use Committee (IACUC) in compliance with the Association for Assessment and Accreditation of Laboratory Animal Care (AAALAC) approved practices.

\section{Behavioral measures}

Neonatal animals (P7-P14) were transferred from their home cage to opaque chambers with a translucent lid and acclimated in a temperature-controlled environment for $10 \mathrm{~min}$ before assessments. Adolescent animals $(\geq$ P35) were transferred to raised translucent boxes with a grid mesh bottom and acclimated for $25 \mathrm{~min}$ before data collection. After habituation, behavioral experiments followed including spontaneous paw guarding assessment, muscle mechanical withdrawal thresholds, cutaneous mechanical withdrawal thresholds, grip strength and/or proprioceptive behaviors. No cohort received more than two of the listed behaviors at a time to reduce stress and maternal separation time for neonatal animals. Data were obtained at baseline (BL), $1 \mathrm{~d}$ and/or $3 \mathrm{~d}$, or up to $21 \mathrm{~d}$ for adolescent animals, postinjury as indicated.

Spontaneous paw guarding assessments scores preferential weight bearing on a scale of zero to two, where zero is no guarding after injury, 1 indicates shifted weight bearing but the paw still touches the floor and 2 indicates full paw lifting. Assessments were made for a duration of $1 \mathrm{~min}$. Neonatal animals were scored every $5 \mathrm{~min}$ for $30 \mathrm{~min}$, and adolescent animals were scored for $1 \mathrm{~h}$.

Hind paw muscle withdrawal was assessed with a digital Randall Selitto device (IITC Life Science Inc.) with a dulled probe attachment $\sim 2 \mathrm{~mm}$ wide at the tip. The dorsal paw was supported by the upper machine arm and the medial plantar paw was slowly pressed with the dulled probe until a robust withdrawal response was evoked. The gram force that elicited a withdrawal response was considered threshold. Three trials were obtained in 5-min (minimum) intervals and averaged together for analysis. Maximum squeezing force was $150 \mathrm{~g}$ for neonates and 350 or $500 \mathrm{~g}$ for adolescent mice of different strains.

Cutaneous mechanical withdrawal thresholds were assessed on the dorsal surface of the hindpaw as described in previous reports (Marsh et al., 1999; Jankowski et al., 2014; Liu et al., 2017) for neonatal animals using an increasing series of calibrated von Frey filaments ranging from 0.07 to $6 \mathrm{~g}$. Threshold to withdrawal was determined in three trials with 5-min intervals between trials and averaged.

Dynamic paw muscle strength was assessed by neonatal hanging time (Feather-Schussler and Ferguson, 2016). Animals were held with forepaws near a thin metal rod spanning a $9.5 \mathrm{~cm}$ in diameter apparatus until the rod was gripped. Animals were timed while freely hanging above a $12-\mathrm{cm}$ padded drop until they released the bar, escaped the apparatus by climbing out, or $60 \mathrm{~s}$ was reached. An escape was determined to require enough muscle strength to pull up and out of the apparatus and was thus set to maximum time. Three trials were recorded and averaged.

To evaluate proprioception, we recorded the animals' innate righting reflex (Dallman and Ladle, 2013). Animals were gently turned over and placed on their back. The time to flip over to all four limbs was recorded and averaged over three trials with 5-min intervals between trials. 
Animals were weighed and their temperature was taken after an injection of GH to determine whether any GH-related side effects were present with our injection strategy; $24 \mathrm{~h}$ after an intramuscular hindpaw injection, animals were weighed on a tabletop scale and their temperature was recorded using a digital surface contact thermometer pressed against the chest of the anesthetized animal according to previous methods (Goodrich, 1977; Liu et al., 2017).

\section{Injections}

GH was injected directly into the hind paw muscles in uninjured mice or in animals receiving incision injury unless otherwise noted in the figure legend. Dosing ranged from 0.1 to $1.5 \mathrm{mg} / \mathrm{kg}$ in $10 \mu \mathrm{l}$ for all neonatal experiments. Adolescent animals were injected with the $1.5 \mathrm{mg} / \mathrm{kg}$ dose in $18 \mu \mathrm{l} ; 5 \mathrm{mg} / \mathrm{kg} \mathrm{GH}$ binding protein (GHBP) or vehicle $(0.1 \%$ bovine serum albumin) in PBS was injected in $10 \mu$ into the hindpaw muscle. To induce Cre-recombinase in AdvGHr animals, tamoxifen was made fresh at $25 \mathrm{mg} / \mathrm{ml}$ in corn oil and uninjured animals were singly injected intraperitoneally at P7 at a dose of $250 \mathrm{mg} / \mathrm{kg}$ tamoxifen (Hester and Danzer, 2013).

\section{Surgical hind paw incisions}

Animals were anesthetized with $2-3 \%$ isoflurane and a longitudinal incision of the right hairy hind paw skin was made lateral to the main saphenous nerve innervation territory. Then incision was continued in between the bones through to the flexor digitorum brevis (FDB) muscles. Blunt manipulation of the muscle was performed using \#5 forceps, but the plantar skin was left untouched. Before wound closing with 7-0 sutures, interventions corresponding to the experiment were injected into the incision site. When appropriate, adolescent surgical hind paw incisions were performed using the same procedures, with wounds closed with 6-0 sutures. Animals were allowed to recover for the indicated times. For comparisons, some cohorts only received the hairy skin incision or a single suture through intact skin (sham) but did not experience the muscle incision. For dual incision assays, similar procedures were followed as described above except the first incision was made at P7, and the second incision (when indicated) was performed at P35.

\section{Sciatic nerve injections}

Mice were placed on a warming pad and kept under 2-3\% isoflurane anesthesia as the right sciatic nerve was revealed by a small incision of the skin and cautious separation of the underlying muscle. Carefully, as to not stretch the nerve, the sciatic was separated from surrounding tissue and raised onto a malleable plastic platform. Targeting or control siRNAs were injected directly into the sciatic nerve above the trifurcation using quartz microelectrodes connected to a picospritzer with 8-10 short pulses at 1-2 psi. Approximate volume of injection was $\sim 100 \mathrm{nl}$. Four different duplexes from ON-TARGETplus SMARTpool siRNAs (Dharmacon, Lafayette; catalog \#4390771) against SRF were first tested in vitro (Neuro2a cells) to determine knock-down efficiency of the individual siRNA duplexes similar to previous reports (Queme et al., 2016; Ross et al., 2016; Liu et al., 2017; data not shown). The most efficient sequence was determined using real-time PCR and used for all subsequent in vivo analyses (sense: 5'-S-S-GCAGCAACCUCACCGAG CUUU; antisense: 5'-P-AGCUCGGUGAGGUUGCUGCUU). siRNAs were first conjugated to Penetratin-1 according to manufacturer's instructions after thiol removal (Dharmacon) and reconstituted at 90 $\mu \mathrm{M}$. Depending on age, siRNAs were then injected into the sciatic nerve as described above $1 \mathrm{~d}(<\mathrm{P} 10)$ or $2 \mathrm{~d}(\geq \mathrm{P} 10)$ before incision and further experimentation to allow for retrograde transport of the siRNAs to the DRG somas. The non-coding control siRNA has been used previously and does not target any murine gene (ThermoFisher D-001206-14-05).

\section{Real-time RT-PCR}

RNA was isolated from lumbar 3/4/5 (L3/4/5) dorsal root ganglia (DRGs) on the side ipsilateral to injury. RNeasy Mini kit (QIAGEN stock \#74104) was performed on DRGs for total mRNA isolation and RNeasy Fibrous Tissue Mini kit (QIAGEN stock \#74704) was used to isolate muscle mRNA. All RNA isolations were performed exactly
Table 1. Primer information for standard real-time PCR

\begin{tabular}{|c|c|c|}
\hline \multicolumn{3}{|c|}{ mRNA primers } \\
\hline Gene & Forward & Reverse \\
\hline ASIC3 & ATGAAACCTCCCTCAGGACTGG & AACTCCCCATAGTAGCGAACCC \\
\hline ELK1 & AAGAATTGGAAGCTGCAAGGGCTG & TGTTCTCTGTTAGGATGGCTGGGA \\
\hline ELK3 & ACACACACACAACCAAGATG & TCAGAGCGCTGGGATTATAG \\
\hline ELK4 & GGTGATTTGTGGGAGAGTAG & САССTCCTATCTCTGGGTAT \\
\hline Fcer2a & GATCTAAGGAACGCCCAATC & CTGTGCGCTTCTCATTCA \\
\hline GAPDH & ATGTGTCCGTCGTGGATCTGA & ATGCCTGCTTCACCACCTTCTT \\
\hline GDNF & AGCTGCCAGCCCAGAGAATT & GCACCCCCGATTTTTGC \\
\hline GHr & GCCTCTACACCGATGAGTAA & GGAAAGGACTACACCACCT \\
\hline IGFr1 & TTGAACTTATGCGCATGTGCTGGC & TCTCATCCTTGATGCTGCCGATGA \\
\hline IL1-r & AGGAATGTGGCTGAAGAGCACAGA & ACTCGTGTGACCGGATATTGCTTC \\
\hline IL1 $\beta$ & TACAAGGAGAACCAAGCAAC & GGTGTGCCGTCTTTCATTA \\
\hline MCP1 & САCCTGCTGCTACTCATTC & CTACAGCTTCTTTGGGACAC \\
\hline $\mathrm{NF} \kappa \mathrm{B}$ & CTGCACCAAGACGGAACC & GAGCCTTCTCAAGAAAGAGGTTATC \\
\hline NGF & ACACTCTGATCACTGCGTTTTTG & CCTTCTGGGACATTGCTATCTGT \\
\hline OSMr & TCCAGGCTCACCCTTATT & AGCCTCGGTGTGTAGTT \\
\hline $\mathrm{P} 2 \times 3$ & ACAAGATGGAGAATGGCAGCGAGT & TGATGTTGAACTTGCCAGCGTTCC \\
\hline P2Y1 & GATGAATTTGCGAGCACGGTTGGA & TCCACACAGCTGTTGAGACTTGCT \\
\hline SRF & TGGAGTTCATCGACAACAAG & AGCGTGGACAGCTCATA \\
\hline STAT1 & сCCAGGAATCTCTCCTTCTT & GACCTCTCTTGGTGACTGAT \\
\hline STAT3 & CTGGGTCTGGCTAGACAATA & CGCTCCTTGCTGATGAAA \\
\hline STAT5 & CCCACGTCAGTTGTAGTATC & GTTCAGCTCTTACACGAGAG \\
\hline TNFa-r & TCGGAAAGAAATGTCCCAGGTGGA & TGGAACTGGTTCTCCTTACAGCCA \\
\hline $\operatorname{TNF} \alpha$ & сCTATGTCTCAGCCTCTTCT & GGGAACTTCTCATCCCTTTG \\
\hline TRPA1 & GCAGGTGGAACTTCATACCAACT & CACTTTGCGTAAGTACCAGAGTGG \\
\hline TRPV1 & TTCCTGCAGAAGAGCAAGAAGC & CCCATTGTGCAGATTGAGCAT \\
\hline
\end{tabular}

Included are all sequences used in Table 3 and other figures or text that contain PCR data.

according to the manufactures' directions. For standard real-time PCR assessments, $500 \mathrm{ng}$ of total RNA was reverse transcribed into cDNA and real-time PCR was performed using SYBR Green Master Mix on a StepOne real-time PCR system (Applied Biosystems). Quantitative PCR was analyzed by the $\Delta \Delta$ cycle threshold (CT) method with normalization to GAPDH. Differences in expression are determined from the normalized $\Delta \Delta \mathrm{Ct}$ values and standard error of the difference in means is determined. This was used to calculate fold change between conditions and values are then converted to a percent change where 2 -fold $=100 \%$ change (Queme et al., 2020). Primer sequences are all recorded in Table 1.

\section{Western blotting}

FDB muscle or L3/4/5 DRGs were dissected and frozen on dry ice. After homogenization in protein lysis buffer as completed previously (Ross et al., 2018), 20- $\mu$ g samples were boiled in gel loading buffer containing $\beta$-mercaptoethanol as a reducing agent and loaded onto a $12 \%$ or “AnyKD" precast polyacrylamide gel (Bio-Rad 4569033) for Western blot analysis. Gels were transferred to a polyvinylidene difluoride membranes (PVDF; Merck Millipore Ltd.) at $35 \mathrm{~V}$ for $16-18 \mathrm{~h}$ at $4^{\circ} \mathrm{C}$. The next day, transfer quality was assessed by staining (Coomassie Brilliant Blue Bio-Rad 1610436) the gel for any remaining proteins. The membrane was washed, blocked with Odyssey blocking buffer (BB; LiCor 927-40000) diluted in PBS (1:4), and incubated in $2 \times$ PBS with $0.2 \%$ Tween and BB (1:1) with primary antibodies. After incubation overnight, the membranes were washed, incubated in $2 \times$ PBS with $0.2 \%$ Tween and $0.01 \%$ SDS and BB (1:4) with appropriate infrared-conjugated secondary antibodies (LiCor) and visualized on LiCor Odyssey CLx protein imaging system. Exposure times were consistent between runs and gain was always set to 1.0. Band intensity was then quantified using ImageJ software (NIH) similar to previous procedures (Liu et al., 2017). Primary antibodies included GH (LS-C146263, polyclonal rabbit, 1:1000) and GAPDH (Abcam 83956, polyclonal chicken, 1:2000).

\section{Immunohistochemistry (IHC)}

DRGs, sciatic nerve and hindpaw muscle were sectioned on a cryostat at $10 \mu \mathrm{m}$ (DRG and sciatic nerve) or $20 \mu \mathrm{m}$ (muscle). DRGs were frozen on 
dry ice in OCT medium and muscle was snap frozen in liquid nitrogen. All tissue was kept at $-80^{\circ} \mathrm{C}$ until use. Cryostat sections were then fixed on the slide. Slides were washed and blocked before overnight primary antibody incubation. The next day, the tissue was washed and stained for secondary antibodies before cover slipping with mounting media containing DAPI to mark nuclei (Fisher Scientific 17985-50). For immunocytochemistry, samples were processed by Cincinnati Children's Pathologic core. Briefly, slides were pretreated with citrate buffer, washed and incubated with primary antibody for $32 \mathrm{~min}$. Detection was completed with the 3,3'-diaminobenzidine (DAB) rabbit kit (Ventana $\# 760$-151), counterstained with hematoxylin and blued with bluing reagent, and then dehydrated before coverslipping. Primary antibodies used were: GH [LS-C146263, polyclonal rabbit, 1:500 (fluorescence) or 1:100 (DAB)], GHr (Abcam 202964, polyclonal rabbit, 1:1000), SRF (Abcam 53147, polyclonal rabbit, 1:250), and dystrophin (Abcam 15277, polyclonal rabbit, 1:250). Fluorescent imaging was observed on a Nikon confocal microscope and all gain and laser power were maintained equally across all samples in each experiment. For quantification, images were converted to grayscale by a blind investigator and an equal threshold was applied to all images. A $200 \times 200$ region of interest (ROI) defined by positive staining was analyzed for particles of sufficient size and were quantified for mean gray value (ImageJ User Guide).

To quantify myofiber cross sectional area, we used the protocol established by Nikolaou and colleagues (Nikolaou et al., 2015). Briefly, dystrophin-stained sections of hindpaw muscle were acquired on the Nikon confocal microscope at high intensity to obtain consistent signal around myofibers. Images were captured using NIS Elements software and then using Fiji software (Schindelin et al., 2012), images were converted to eight-bit binary images. Converted images were then manually edited to remove non-muscle regions or damaged fiber staining. Myofiber cross-sectional area measurements were then obtained for each fiber in the section. Three non-consecutive sections per condition were analyzed and averaged.

\section{Ex vivo preparation}

A novel neonatal ex vivo hind paw muscle-tibial nerve-DRG-spinal cord recording preparation was used to directly assess the response properties of individual primary afferent neurons under our various conditions. Briefly, based on the forepaw prep previously described (Queme et al., 2020), animals were first anesthetized with a mix of ketamine and xylazine (100 and $16 \mathrm{mg} / \mathrm{kg}$, respectively) and then perfused with ice-cold oxygenated $\left(95 \% \mathrm{O}_{2} / 5 \% \mathrm{CO}_{2}\right)$ artificial CSF (aCSF; $127.0 \mathrm{~mm} \mathrm{NaCl}, 1.9$ mм KCl, $1.2 \mathrm{~mm} \mathrm{KH}_{2} \mathrm{PO}_{4}, 1.3 \mathrm{~mm} \mathrm{MgSO}_{4}, 2.4 \mathrm{~mm} \mathrm{CaCl}_{2}, 26.0 \mathrm{~mm}$ $\mathrm{NaHCO}_{3}$, and $10.0 \mathrm{~mm}$ D-glucose). The intact spine and right hind leg were isolated and transferred to a new dish with circulating oxygenated aCSF. The hind paw muscle (with bone intact), tibial/sciatic nerve, L1L6 DRGs and corresponding spinal cord segments were dissected in continuity. The spinal cord was hemisected, and the intact preparation was then transferred to a new recording chamber under the same conditions. The paw with revealed muscle was pinned to a metal grate within an inner bath under its own circulation of $\mathrm{O}_{2}$ aCSF. The nerve was fed through a small gap of the inner bath and the spinal cord and DRGs were pinned within the outer dish. The hole between the dishes was filled with petroleum jelly to separate the baths and hold the nerve in place. The bath was slowly warmed to $32^{\circ} \mathrm{C}$.

Quartz microelectrodes (impedance $>150 \mathrm{M} \Omega$ ) containing $5 \%$ Neurobiotin (Vector Laboratories) in $1 \mathrm{~m}$ potassium acetate were used for sharp electrode single unit recordings in the L3 or L4 DRGs. An impaled cell body was determined to have axons in the tibial nerve by a response from electrical search stimulus by a suction electrode placed on the side of the nerve. Once a cell was determined to have axons in the tibial nerve, the muscle was probed with concentric bipolar electrode to locate the cell's receptive field (RF) in the hindpaw muscle. Then the muscle was probed with mechanical stimuli, thermal stimuli and chemical stimuli in this order. For mechanical stimuli, an increasing series of von Frey filaments ranging from $0.07-10 \mathrm{~g}$ was used to stimulate the RF for $\sim 1-2 \mathrm{~s}$. Then cold $\left(\sim 2^{\circ} \mathrm{C}\right)$ followed by hot $\left(\sim 53^{\circ} \mathrm{C}\right)$ physiological saline was delivered to the RF. Following the thermal stimulations, two distinct metabolite mixtures were slowly introduced into the inner bath surrounding the hind paw muscles. First a "low" concentration of metabolites ( $15 \mathrm{~mm}$ lactate, $1 \mu \mathrm{M} \mathrm{ATP}, \mathrm{pH} 7.0$ ) were applied for $\sim 2 \mathrm{~min}$ and then washed out. After washout, a "high" concentration of metabolites ( $50 \mathrm{~mm}$ lactate, $5 \mu \mathrm{M}$ ATP, $\mathrm{pH}$ 6.6) was added to the inner chamber in the same manner. Metabolites were oxygenated and heated to physiological conditions with an in-line heater to maintain bath conditions. ATP was added just before perfusion of the muscle. After metabolite stimulation, mechanical and thermal responsiveness was again assessed.

All activity was recorded by Spike2 software (Cambridge Electronic Design) and was later analyzed offline. Response latencies were recorded and divided by nerve length to determine conductance velocities to categorize Group IV afferents $(\leq 1.2 \mathrm{~m} / \mathrm{s})$ or Group III afferents $(1.2-14 \mathrm{~m} /$ s). Mechanical thresholds were determined by the least amount of force necessary to elicit at least two action potentials. Peak instantaneous frequencies (IF) were determined to assess the maximum response to a peripheral stimulus while firing rates (FR) were determined to obtain the maximum number of events that occurred over a given period of time (200-ms bins). The distribution analyses were determined to be the number of cells that responded to a given stimuli divided by the number of total cells receiving that stimulus. Since chemical stimulation was not given to every cell tested, the distribution of each subtype of chemically activated cells (low, high, or both) was divided by total chemically responsive cells per condition. No differences in response properties were found between cells obtained at the beginning of a recording experiment compared with the end of the session. Based on previous work (Jankowski et al., 2014; Queme et al., 2020) and power analyses, we determined that $\geq 50$ cells per group would be required.

\section{Experimental design and statistical analysis}

Data were analyzed using SigmaPlot software (v.14). Critical significance was set to $\alpha<0.05$. All data were first checked for normal distribution with Shapiro-Wilk and equal variance with Brown-Forsythe and then parametric or nonparametric tests were used accordingly. Specific tests are indicated in the figure legends. For behavioral data containing the same animals treated with an intervention over time, a two-way repeated measures (RM) ANOVA was used. In behavioral data in which only one time point or a percent change from $\mathrm{BL} /$ naive was compared across groups a one-way ANOVA was used. Analysis of cDNA, protein quantification, area under the curve, or ex vivo analysis was measured by a one-way ANOVA or corresponding non-parametric test across groups. All analyses that passed the omnibus test were further discriminated by Tukey's, Holm-Sidak, or Dunn's post hoc test analysis as noted in figure legends. Discrete categorical data were analyzed by $\chi^{2}$ across all groups. Graphical panels were made using GraphPad Prism (v.8) and compiled in Adobe Photoshop. In all studies involving subjective measures including all behavior, ex vivo, and IHC quantification, the researcher was blinded by co-investigators or by the unknown genotype of the animal. The rare occasion (as marked in figure legends) of a detected outlier defined as being $>2$ SDs away from the mean was removed. Biological replicates $(n)$ are provided for each figure in the legends or text. Technical replicates are explained above for each experiment.

\section{Results}

\section{Sensory neuron-specific deletion of GHr modulates} behavioral phenotypes in uninjured neonatal mice

Previously, we have demonstrated that a systemic reduction in GH levels resulted in neonatal hypersensitivity (P7-P14) to peripheral stimuli that resolved by P21 (Ford et al., 2019). To determine whether the effects of reduced GH-signaling on nociception were peripherally mediated, we injected GHBP locally into the right hind paw of uninjured neonatal (P7-P14) mice: the time frame in which we observed GH deficiency-related hypersensitivity. We found that $1 \mathrm{~d}$ after injection, GHBP resulted in spontaneous paw guarding behaviors (Fig. 1A). To then test whether the anti-nociceptive effects of $\mathrm{GH}$ were because of a direct effect on primary afferents, we developed a transgenic mouse that allowed for targeted deletion of $\mathrm{GHr}$ in a time 
A

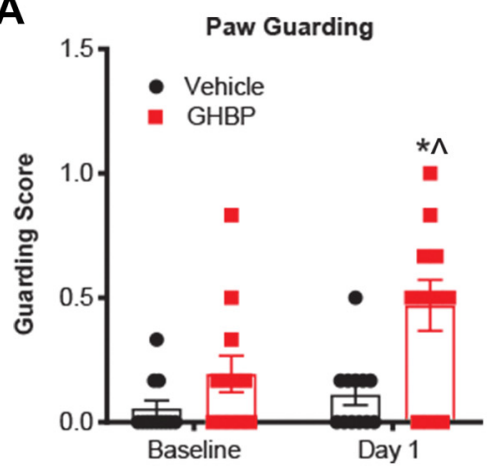

C

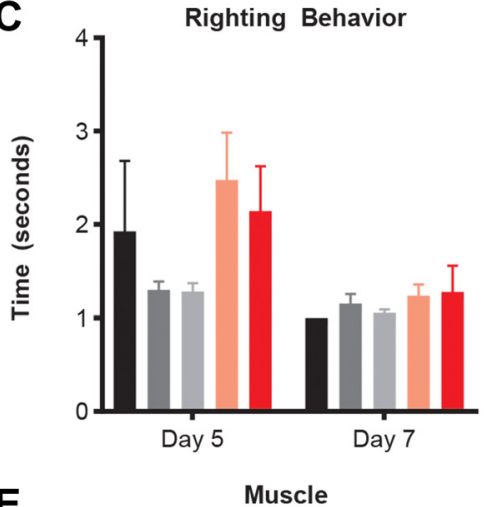

B

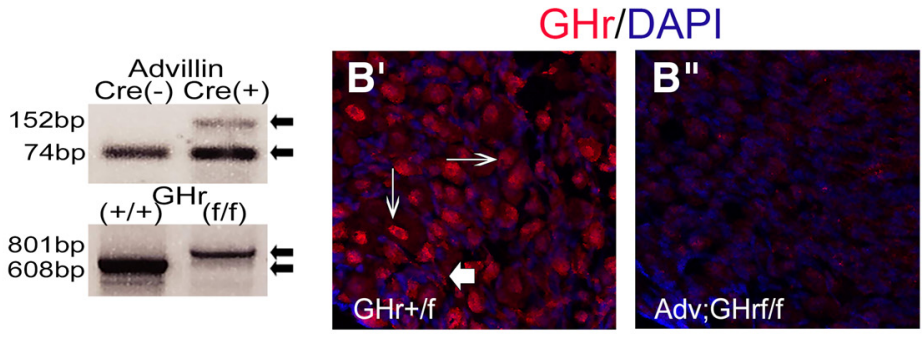

Cutaneous

D

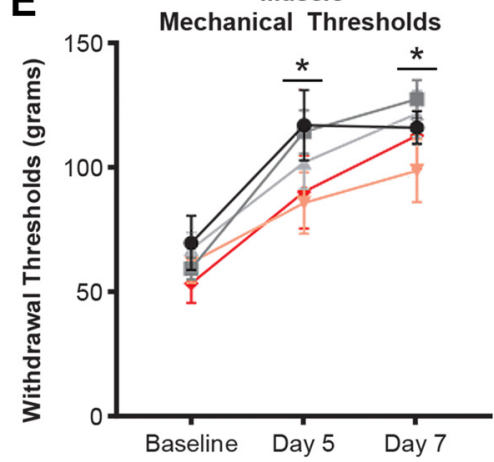

E'

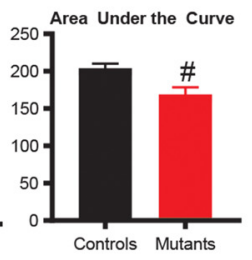

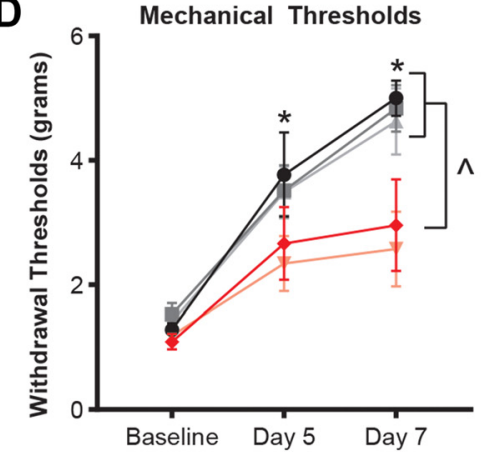

Dynamic

$\mathbf{F}$

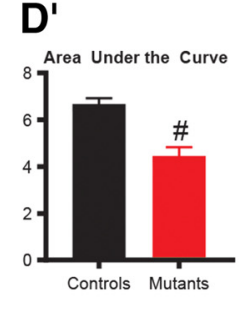

Muscle Strength

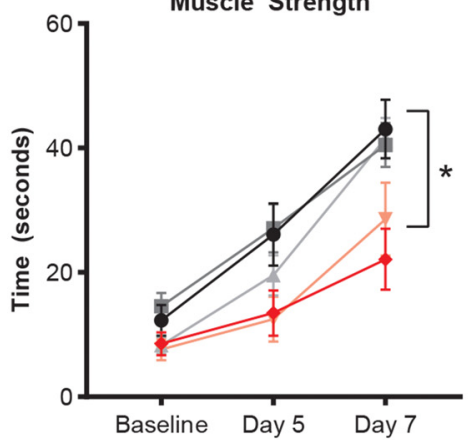

$\mathbf{F}$

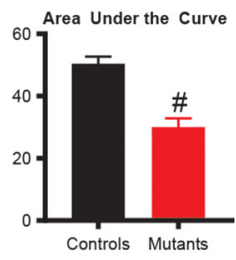

$\longrightarrow$ WT $\longrightarrow \mathrm{GHr}(+/ \mathrm{f}) \longrightarrow \mathrm{GHr}(\mathrm{f} / \mathrm{f})$
$\longrightarrow \mathrm{Adv} ; \mathrm{GHr}(+/ \mathrm{f}) \longrightarrow \mathrm{Adv} ; \mathrm{GHr}(\mathrm{f} / \mathrm{f})$

Figure 1. Modulation of neonatal nociception by peripheral GH signaling. $A$, In GHBP experiments, we saw overall effects of injection $\left(F_{(1,21)}=4.68, p=0.042\right)$ and day $\left(F_{(1,21)}=19.6, p<0.001\right)$, and a moderate interaction $\left(F_{(1,21)}=3.63, p=0.071\right)$. After Tukey's correction, we determined adjusted $p$ values that demonstrated that there is no difference between groups at BL $(p=0.499)$ but there is at day $1\left({ }^{\wedge} p=0.007\right.$ vs controls). Also, we found no effect of day in our control group $(p=0.082)$, but we did see a significant effect in GHBP injected animals at day 1 ( $* p=0.001 \mathrm{vs} \mathrm{BL);} n=12 /$ group, two-way RM ANOVA, Tukey's post hoc test. $\boldsymbol{B}$, Representative genotyping analysis of Adv-Cre; $\mathrm{GHr}^{\mathrm{f} / \mathrm{f}}$ mice. $\boldsymbol{B}^{\prime}, \boldsymbol{B}^{\prime \prime}$, Immunostaining of DRGs for GHr (red) and nuclear labeling using DAPI (blue) in tamoxifen treated control (Adv; $\mathrm{GHr}^{+/ f}$ ) and Adv; GHr/f $\mathrm{mice}^{\mathrm{f}}$. Arrows indicate $\mathrm{GHr}+$ neuronal staining, large arrow indicates nerve fiber. $C$, Righting reflexes are not different across groups at 5 and $7 \mathrm{~d}$ after tamoxifen (main effect of genotype $F_{(4,156)}=$ $2.95, p=0.024)$. Adjusted $p$ values indicate no differences between groups at day 5 ( $p=0.991$ and enclosed) and day $7(p=0.913$ and enclosed); $n=11-14$ (GHr $+/+), 26-30$ $(G H r f /+), 26-29(G H r f / f), 13-16(A d v ; G H r f /+)$, and 5-7 (Adv;GHrf/f)/time point, two-way RM ANOVA, Tukey's post hoc test. D, Cutaneous mechanical withdrawal thresholds behaviors demonstrated an effect of day $\left(F_{(2,91)}=64.05, p<0.001\right)$, genotype $\left(F_{(4,91)}=3.86, p=0.007\right)$, and an interaction $\left(F_{(2,91)}=2.19, p=0.035\right)$. After Tukey's correction, adjusted $p$ values showed an increase over time in control groups but not in $\mathrm{Adv} ; \mathrm{GHr}^{\mathrm{f} /+}\left(n=8-12 /\right.$ time point) nor $\mathrm{Adv} ; \mathrm{GHr}{ }^{\mathrm{f} / \mathrm{f}}$ (8-10/time point) animals; $* p<0.001$ versus $\mathrm{BL}$; $\left({ }^{\wedge} p<0.05\right.$ versus each control group. Two-way RM ANOVA, Tukey's post hoc test. $D^{\prime}$, Combined mutant $\left(\mathrm{Adv} ; \mathrm{GHr}^{\mathrm{f} /+}, \mathrm{Adv} ; \mathrm{GHH}^{\mathrm{f} / \mathrm{f}}\right)$ groups have significantly lower cutaneous mechanical withdrawal thresholds compared with control groups $\left(\mathrm{GHr}^{+/+}, \mathrm{GHr}^{\mathrm{f} /+}, \mathrm{GHr}^{\mathrm{f} / \mathrm{f}}, n=6-7,16-22,10-14 /\right.$ time point, respectively) in an area under the curve from BL (P7) to $7 \mathrm{~d}$ (P14) post-tamoxifen; $F_{(1,46)}=24.39$, \#p $<0.001$ versus controls. One-way ANOVA, Tukey's post hoc test. $\boldsymbol{E}$, In the same animals that received cutaneous stimulation, we saw an overall effect of day $\left(F_{(2,91)}=36.6, p<0.001\right)$ in their muscle mechanical thresholds. Tukey's correction showed an increase in threshold over time in all groups; $* p<0.001$ versus BL, two-way RM ANOVA, Tukey's post hoc test. $E^{\prime}$, Area under the curve analysis for muscle mechanical thresholds in combined groups indicates a reduced threshold in GHr mutant groups compared with controls. $F_{(1,47)}=10.76, \# p=0.002$ versus controls. One-way ANOVA, Tukey's post hoc test. $F$, Dynamic muscle strength indicated an overall effect of day $\left(F_{(2,156)}=42.53, p<0.001\right)$ and genotype $\left(F_{(4,156)}=3.88, p=0.006\right)$. Adjusted $p$ values determined by Tukey's post hoc test showed that all groups except $\mathrm{Adv} ; \mathrm{GHr} \mathrm{r}^{\mathrm{f} / \mathrm{f}}$ increased over time by $7 \mathrm{~d}$ posttamoxifen; $* p<0.001$ versus $\mathrm{BL} ; n=$ same as $\boldsymbol{C}$. $\boldsymbol{F}^{\prime}$, Combined group area under the curve analysis reveals significantly reduced time in mutant GHr groups ( $n=18-23 /$ time point) compared with control groups ( $n=65-70 /$ time point). $F_{(1,90)}=28.17$, \#p $<0.001$ versus controls, one-way ANOVA, Tukey's post hoc test. Data shown as mean \pm SEM. 
A

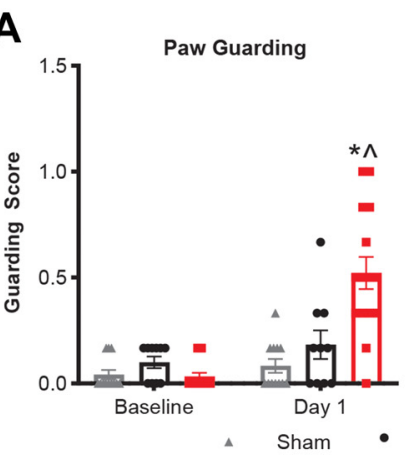

B

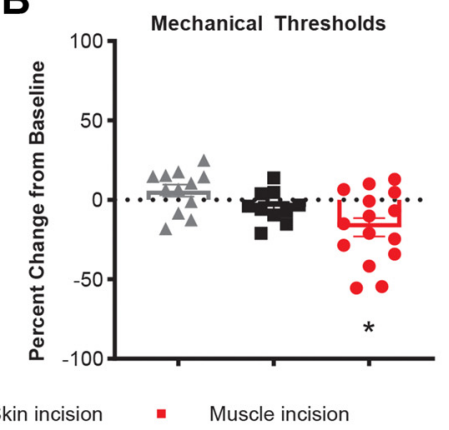

C

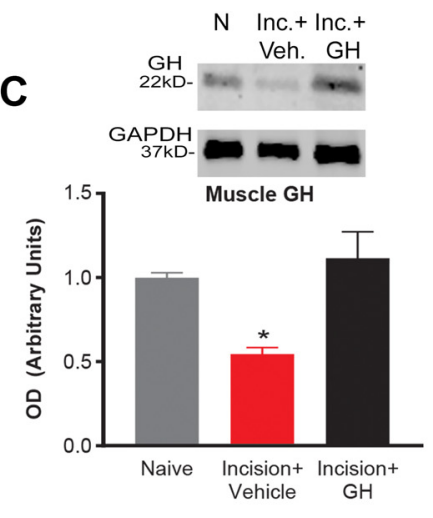

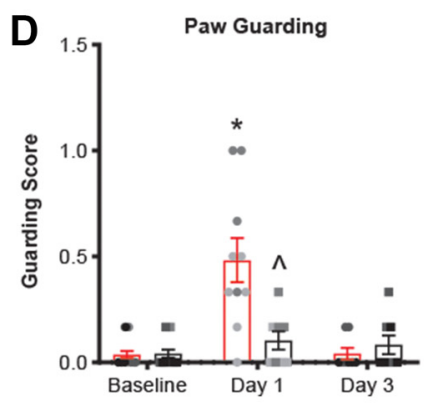
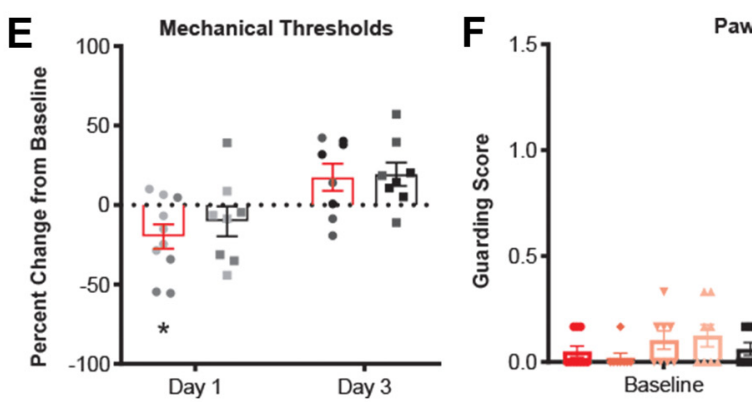

Paw Guarding
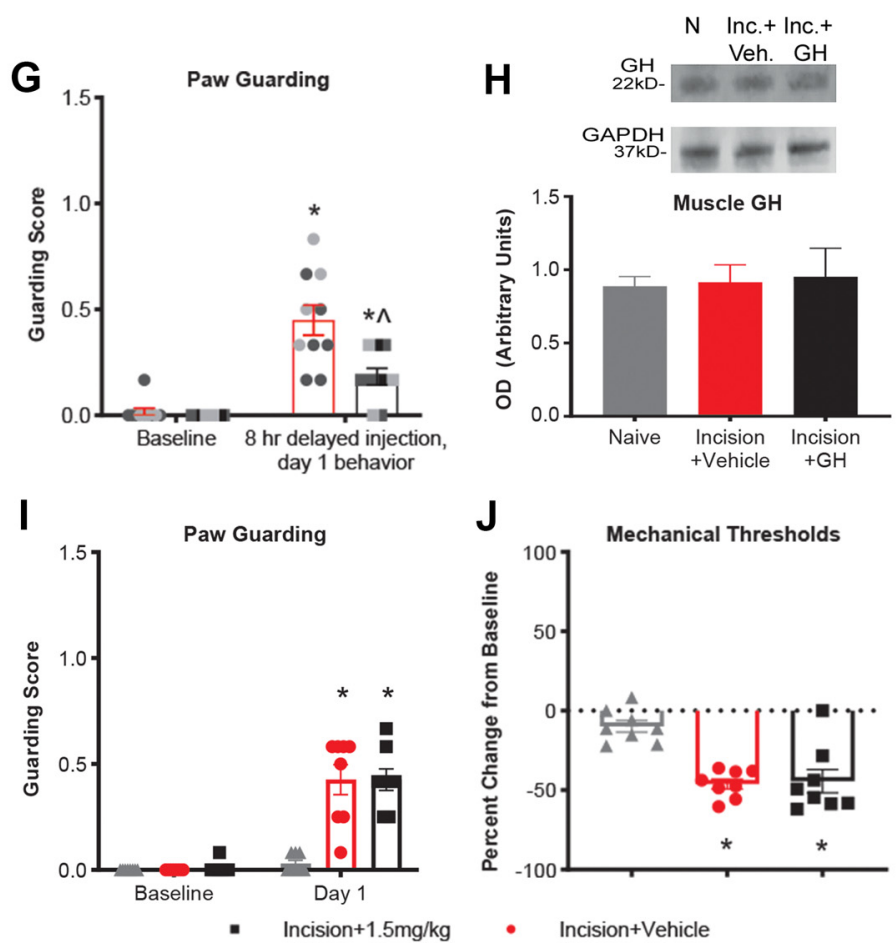

Figure 2. Neonatal animals display pain-like behaviors following a muscle incision that can be prevented by local GH administration. $A$, One day after a reverse neonatal muscle incision, animals guard the injured hindpaw demonstrating an overall effect of day $\left(F_{(1,34)}=23.16, p<0.001\right)$, injury $\left(F_{(2,34)}=12.74, p<0.001\right)$, and an interaction $\left(F_{(2,34)}=12.64, p<0.001\right)$. Tukey's post hoc test showed that animals that received a muscle incision guard at day $1(* p<0.001 \mathrm{vs} B \mathrm{~L})$, while those that receive a skin incision only $(p=0.309)$ or sham $(p=0.575)$ do not. Also, muscle incised animals guard significantly more compared with both controls ( ${ }^{\wedge} p<0.001$ vs controls). $\boldsymbol{B}$, Mechanical withdrawal thresholds in the same animals demonstrated an overall effect of day $\left(F_{(1,34)}=5.2, p=0.029\right)$, injury $\left(F_{(2,34)}=7.28, p=0.002\right)$, and an interaction $\left(F_{(2,34)}=4.52, p=0.018\right)$. Only animals that received a muscle incision displayed lower squeezing withdrawal thresholds compared with BL ( $* p<0.001$ vs BL); $n=10-15 /$ group, two-way RM ANOVA, Tukey's post hoc test. C, Representative image and analysis of muscle GH levels using Western blotting. Following incision, GH levels are reduced in incised animals compared with naive animals and this is restored with exogenous $\mathrm{GH}$ treatment. $F_{(2,6)}=9.96, * p=0.012$ versus naive; $n=3$ /group, one-way ANOVA, Tukey's post hoc test. $\boldsymbol{D}$, Spontaneous paw guarding is increased following incision in animals treated with vehicle but not in GH-treated animals at $1 \mathrm{~d}$. By $3 d$, neither group is different from their BL or each other. Overall effect of day $\left(F_{(1,16)}=13.79, p=0.002\right)$, injury $\left(F_{(1,16)}=7.89, p=0.013\right)$, and interaction $\left(F_{(1,16)}=9.37, p=0.007\right)$. Tukey's post hoc test indicated that vehicle-injected animals guard at day $1(* p<0.001 \mathrm{vs} \mathrm{BL}$ ), while those injected with $1.5 \mathrm{mg} / \mathrm{kg}$ of $\mathrm{GH}$ do not $(p=0.668)$. There was also a significant difference between groups at day $1\left({ }^{\wedge} p<0.001\right.$ vs controls); $n=8-10 /$ group, two-way RM ANOVA. The shades of the data points indicate the age range of the animals (also true for $\boldsymbol{E}$, $\boldsymbol{G}$ ). 
dependent manner in sensory neurons. We crossed the AdvcreERT2 mouse (Lau et al., 2011) with a floxed GHr line and injected neonatal pups with tamoxifen at P7 to initiate sensory neuron-specific deletion of GHr (Fig. 1B). Using this strategy, we first confirmed that control animals expressed normal levels of $\mathrm{GHr}$ in the DRGs while tamoxifen injected Adv; $\mathrm{GHr}^{\mathrm{f} / \mathrm{f}}$ neonates had significantly reduced $\mathrm{GHr}$ expression as assessed with immunocytochemistry (Fig. 1B', B'). Real-time PCR analysis confirmed that within 5-7 d posttamoxifen injection, that $\mathrm{GHr}$ mRNA was significantly reduced in the DRGs compared with controls $\left(-45 \pm 25 \%\right.$ vs controls; $F_{(1,15)}=4.54$, *p $p=0.039 ; n=5-$ 13 per group). This corresponded with an upregulation of insulin-like growth factor receptor 1 (IGFr1; $136 \pm 46 \%$ vs controls $(H=6.25,1 \mathrm{df}, * p=0.012)$ as well as SRF mRNA $(229 \pm 52 \%$ vs control; $F_{(1,17)}=4.93, * p=0.04$, Tukey's test) confirming previous literature (Carter-Su et al., 2016) that these factors are downstream of GH signaling. Interestingly, STAT5 was not altered in the DRGs of tamoxifen injected Adv; $\mathrm{GHr}^{\mathrm{f} / \mathrm{f}}$ mice $(7 \pm 41 \%$ vs control; $\left.F_{(1,15)}=0.03, p=0.865\right)$.

We then performed behavioral analyses in these animals and found that although proprioception (righting reflexes) was unaffected by the sensory neuron targeted knock out of GHr (Fig. $1 C$ ), we detected alterations in other behaviors. Static cutaneous mechanical responsiveness (von Frey filament withdrawal thresholds) was significantly altered by GHr knock-out in uninjured animals confirming our previous reports that $\mathrm{GH}$ signaling has important regulation of cutaneous nociception in neonates (Fig. 1D; Liu et al., 2017). We then extended the cutaneous evaluation in this study by also analyzing muscle-related effects. Sensory neuron GHr mutant animals display slight differences when the muscles are probed (muscle squeezing) over time (Fig. $1 E$ ). Further, in a dynamic neonatal muscle strength assay, we

$\leftarrow$

Light gray $=\leq P 7$. Medium gray $=P 8$ and P9. Dark gray $=P 10, P 11$. Black $=\geq P 12 . E$, Muscle withdrawal thresholds are reduced in incised animals with vehicle injection at $1 \mathrm{~d}$, but this is inhibited in GH-treated animals. By $3 \mathrm{~d}$, both groups have increased withdrawal thresholds compared with BL. Overall effect of day $\left(F_{(1,16)}=8.16, p=0.011\right)$. Multiple comparisons indicate that vehicle-injected animals have reduced thresholds compared with $B \mathrm{~L}$ $(* p=0.015$ vs BL) but GH-injected animals do not $(p=0.186) ; n=8-10 /$ group, two-way ANOVA, Tukey's post hoc test. $\boldsymbol{F}$, One day after an injury, animals dosed with $\mathrm{GH}$ at $0,0.1$, 0.5 , and $1.0 \mathrm{mg} / \mathrm{kg}$ guarding showed an overall effect of day $\left(F_{(1,37)}=53.64, p<0.001\right)$, a modest effect of injection $\left(F_{(4,37)}=2.199, p=0.088\right)$ and an interaction $\left(F_{(4,37)}=3.71\right.$, $p=0.012)$. Tukey's correction indicated that all groups significantly guarded $(* p<0.05$ vs $\mathrm{BL}$ ) except animals injected with $1.5 \mathrm{mg} / \mathrm{kg}$ of $\mathrm{GH}(p=0.629)$. Also, animals injected with $1.5 \mathrm{mg} / \mathrm{kg}$ had significantly lower guarding scores compared with vehicle-injected animals at $1 \mathrm{~d}(\wedge p<0.001$ vs controls). $\mathbf{G}$, When $\mathrm{GH}$ treatment is delayed $8 \mathrm{~h}$ following the injury an overall effect of day $\left(F_{(1,17)}=42.89, p<0.001\right)$ and injection $\left(F_{(1,17)}=5.35, p=0.033\right)$ with a moderate interaction $\left(F_{(1,17)}=4.01, p=0.062\right)$ was detected. Tukey's correction demonstrated adjusted $p$ values indicating a difference in each group from $B L(* p=0.004$ within $G H$ vs $B L ; * p<0.001$ within vehicle vs $B L$ ). Also, within day 1 , there is a significant reduction in the amount of guarding in GH-injected animals ( $\wedge=0.005$ vs controls); $n=10$ / group, two-way RM ANOVA, Tukey's post hoc test. $\boldsymbol{H}$, Representative images of Western blottings for $\mathrm{GH}$ in naive, incised, and $\mathrm{GH}$-treated incised adolescent animals. Quantification indicates no differences between groups. $F_{(2,9)}=0.063, p=0.94 ; n=4 /$ group, one-way ANOVA. $I$, Adolescent animals with vehicle+incision or $\mathrm{GH}+$ incision guard have a main effect of day $\left(F_{(1,21)}=95.35, p<0.001\right.$ ), injection (or naive; $F_{(2,21)}=20.2, p<0.001$ ), and interaction $\left(F_{(2,21)}=19.01, p<0.001\right)$. Tukey's post hoc test indicate that both incised groups demonstrating guarding $(* p<0.001$ vs $B \mathrm{~L})$ and do not differ from one another $(p=1) . J$, For muscle mechanical withdrawal thresholds, a main effect of day $\left(F_{(1,21)}=138.97\right.$, $p<0.001$ ), injection (or naive; $\left.F_{(2,21)}=3.55, p=0.047\right)$, and interaction $\left(F_{(2,21)}=17.92\right.$, $p<0.001$ ) was detected. After correction, we found that incised groups had reduced threshold $1 \mathrm{~d}$ after injury ( $* p<0.001$ vs BL and naive) and the incised groups do not differ from one another $(p=0.874) ; n=8 /$ group, two-way RM ANOVA, Tukey's post hoc test. Data shown as the mean \pm SEM. found that, unlike littermate controls, animals with sensory neuron-specific GHr knock-out did not significantly increase in strength over time (Fig. 1F). Area under the curve (AUC) analyses, which provide a simplistic view of the combined measures of time and intensity in our groups, indicate that neonates with $\mathrm{GHr}$ mutations have lower overall scores compared with control animals (Fig. 1D'-F').

\section{Local injection of GH at the time of neonatal muscle incision blocks behavioral hypersensitivity but is not effective in adolescent animals}

Since peripheral GH signaling to neurons appeared to significantly influence nociception during early postnatal development, we wanted to test whether this pathway could also modulate injury-related hypersensitivity in the periphery. In order to assess this, we used a "reverse" hindpaw incision model in which surgical incision of the FDB muscles was obtained from the dorsal side of the foot through the hairy skin. The rationale for performing incision in this manner was to reduce cutaneous injury site effects when assessing muscle-specific pain-related behaviors (muscle squeezing). The surgical model produces robust painrelated hypersensitivity in neonates and also allowed us to deliver $\mathrm{GH}$ directly to the injury site. We first confirmed that a neonatal reverse muscle incision resulted in detectable pain-like phenotypes, while an incision only of the hairy skin did not (Fig. 2A,B) similar to previous work (Brennan et al., 1996; Baccei, 2016). We therefore assessed the levels of $\mathrm{GH}$ in the muscle after incision using Western blotting (Fig. 2C) and found that $1 \mathrm{~d}$ after surgery, muscle GH levels were significantly reduced. This corresponded with the observed spontaneous paw guarding behaviors and muscle mechanical hypersensitivity induced by incision $1 \mathrm{~d}$ later (Fig. 2D,E). To determine whether local injection of GH could blunt incision-related hypersensitivity, we first performed a dose response analysis (Fig. $2 F$ ) based on doses of GH that were insufficient to alter functional levels of systemic IGF-1 (Farris et al., 2007). We confirmed that a single injection of $\mathrm{GH}(1.5 \mathrm{mg} / \mathrm{kg}$ in $10 \mu \mathrm{l})$ into the incision site was able to restore muscle $\mathrm{GH}$ levels similar to naive mice (Fig. $2 C$ ). Importantly, we then found that paw guarding scores and muscle mechanical withdrawal thresholds were normalized to BL levels by this single injection of $\mathrm{GH}$ into the incision site compared with vehicle-injected neonates with incision. By $3 \mathrm{~d}$ following the surgical incision, we no longer detected hypersensitivity in vehicle-injected controls or $\mathrm{GH}$ treated animals (Fig. 2D,E). Interestingly, delayed $\mathrm{GH}$ treatment partially blocked spontaneous paw guarding if given within $8 \mathrm{~h}$ (Fig. 2G) of surgery, possibly indicating a time sensitive application window. As a range of ages were used for these studies, we needed to determine whether there were any age-related effects observed within our groups during this developmental time frame which shows changes in processes such as eye opening, hair production and cutaneous sensory responsiveness (Fitzgerald, 2005; Jankowski et al., 2014; Brust et al., 2015). In analyses evaluating the distribution of ages within the P7-P14 time period, we observed no differences in injury induced nor GH treatment groups (embedded in Fig. 2D,E,G). Analysis indicates an even distribution of data points for each group across ages indicating that no specific age resulted in the effects seen with our interventions or injury.

We then wanted to determine whether the anti-nociceptive effects of GH treatment could be observed in older animals but found that adolescent animals (P35) do not display altered muscle GH levels after an injury. Further, these animals do not show 
A
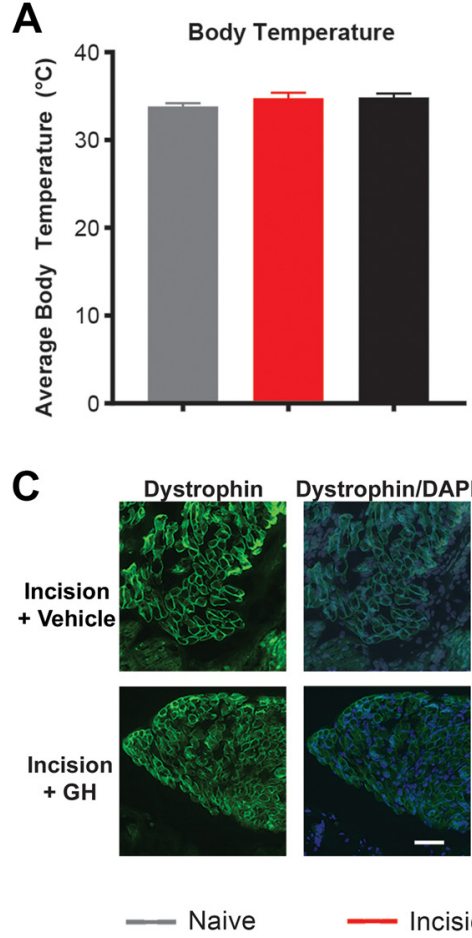

- Incision+Vehicle

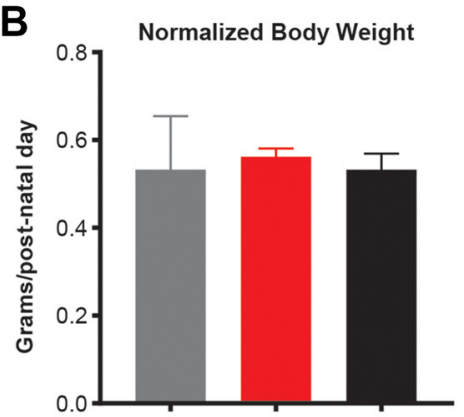

D

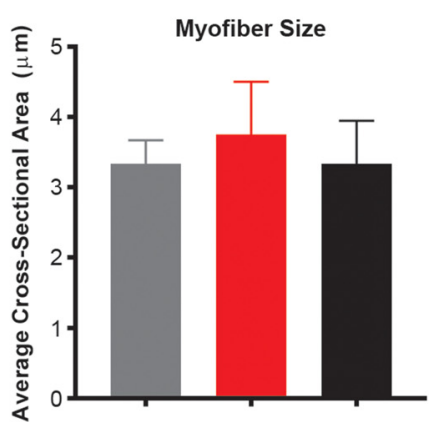

E

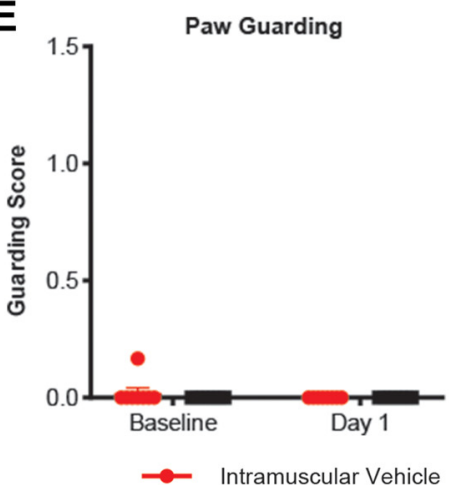

$\mathbf{F}$

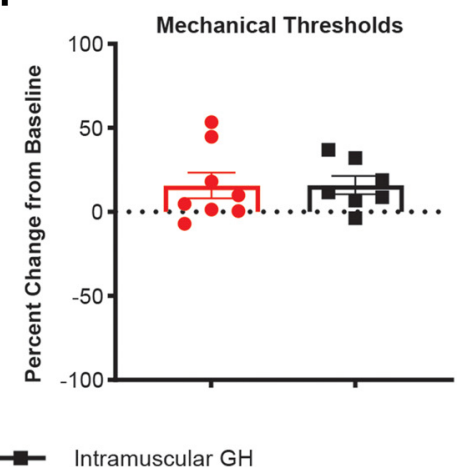

Figure 3. Off-target analyses of local GH injections. $A$, Average body temperature measured on the thorax of neonates was not affected by injury or $\mathrm{GH}$ treatment. $F_{(2,11)}=1.3, p=0.311$. $\boldsymbol{B}$, Body weight normalized to postnatal age was not affected by injury or GH injection. $F_{(2,12)}=0.0754, p=0.928 ; n=4-6 /$ group, one-way ANOVA. $C$, Representative images of myofibers stained with dystrophin (green) in both injured groups. DAPI (blue) co-stain was used to mark nuclei. $\boldsymbol{D}$, The cross sectional area of injured animals was not affected by GH treatment. $F_{(1,4)}=2.24, p=0.209 ; n=3$ animals/group, one-way ANOVA. $E$, Spontaneous paw guarding at day 1 is not affected by local GH $(1.5 \mathrm{mg} / \mathrm{kg})$ injection in mice without incision compared with $\mathrm{BL}\left(F_{(1,13)}=0.867, p=0.369\right)$. $\boldsymbol{F}$, Evoked mechanical withdrawal thresholds are not affected by local GH injection alone compared with vehicle-injected $\left(F_{(1,13)}=0.001, p=0.979\right) ; n=7-8 /$ group, one-way ANOVA. Data shown as mean \pm SEM.

any alterations in pain-related behaviors after muscle incision in response to local GH treatment at this dose (Fig. $2 \mathrm{H}-J$ ). In neonates, we found that the local injection of $\mathrm{GH}$ at the effective dose was not sufficient to alter the cross-sectional area of individual muscle fibers or change physiological conditions of the animals including body temperature and weight. GH injection into uninjured animals also had no effects on BL animal behaviors (Fig. 3). These data suggest that GH can modulate pain-like behaviors specifically in neonates.

Next, we used a novel neonatal ex vivo electrophysiological single unit recording preparation (Fig. $4 A$ ) to assess the response properties of individual primary muscle afferents in mice with incision. We found that the distribution of functional primary muscle afferents was altered by incision. Fewer mechanically sensitive Group III and Group IV muscle afferents $(14 / 50,28 \%)$ were observed in mice with muscle incision plus vehicle injection compared with naive. A similar reduction in the numbers of innocuous metabolite (low responders) responsive units was also observed $(2 / 13,15 \%)$ that corresponded with an increase in the numbers of chemically sensitive cells that responded to both innocuous and noxious (high responders) metabolite mixtures (7/ 13, 54\%). Local GH injection completely reversed the incision-related effects on muscle afferent prevalence (Fig. 4B). We also found that mechanically sensitive muscle sensory neurons had lower thresholds to mechanical deformation of the muscle RFs after incision plus vehicle injection, but animals treated with $\mathrm{GH}$ at the time of the incision did not differ in mechanical thresholds compared with naive (Fig. 4C). $\mathrm{GH}$-treated animals also showed decreased FRs to mechanical stimuli compared with mice with incision although neither group differed from naive (Fig. $4 D, E$ ). These parameters also were not affected by the age in the timeframe we analyzed (embedded in Fig. 4C,D). The FRs of thermally and chemically activated cells were not altered. The mean peak IFs were not statistically altered under any condition in any group (Table 2). Taken together, exogenous local $\mathrm{GH}$ treatment can prevent pain-related behaviors and primary afferent sensitization observed in neonates with muscle incision.

\section{Incision-induced transcriptional} changes in the DRG can be blocked by GH treatment

To determine potential underlying neuronal mechanisms by which $\mathrm{GH}$ mediated anti-nociception after neonatal muscle incision, we analyzed mRNA levels in the DRGs for genes previously found to be altered during neonatal injury (Jankowski et al., 2014) among others known to regulate sensory responsiveness in the periphery in animals $1 \mathrm{~d}$ after injury (see full list in Table 3). Similar to that observed in mice with sensory neuron-specific knock out of the GHr (see above), we found that muscle incision induced a significant increase in IGFr1 mRNA in the DRGs. Also upregulated was the proton sensor and heat transducing channel, TRPV1, and the environmental irritant and cold receptor, TRPA1. ATP sensing ion channel, P2X3 and the proton responsive channel, ASIC3, for example were not altered by incision. Interestingly, $\mathrm{GH}$ treatment at the time of injury blocked the upregulation of all of these factors (Table 3 ).

To assess whether GH could also modulate injury related changes in gene expression in the injured muscles, we then analyzed the expression of candidate cytokines and growth factors known to be altered after injury in the periphery. Muscle incision 

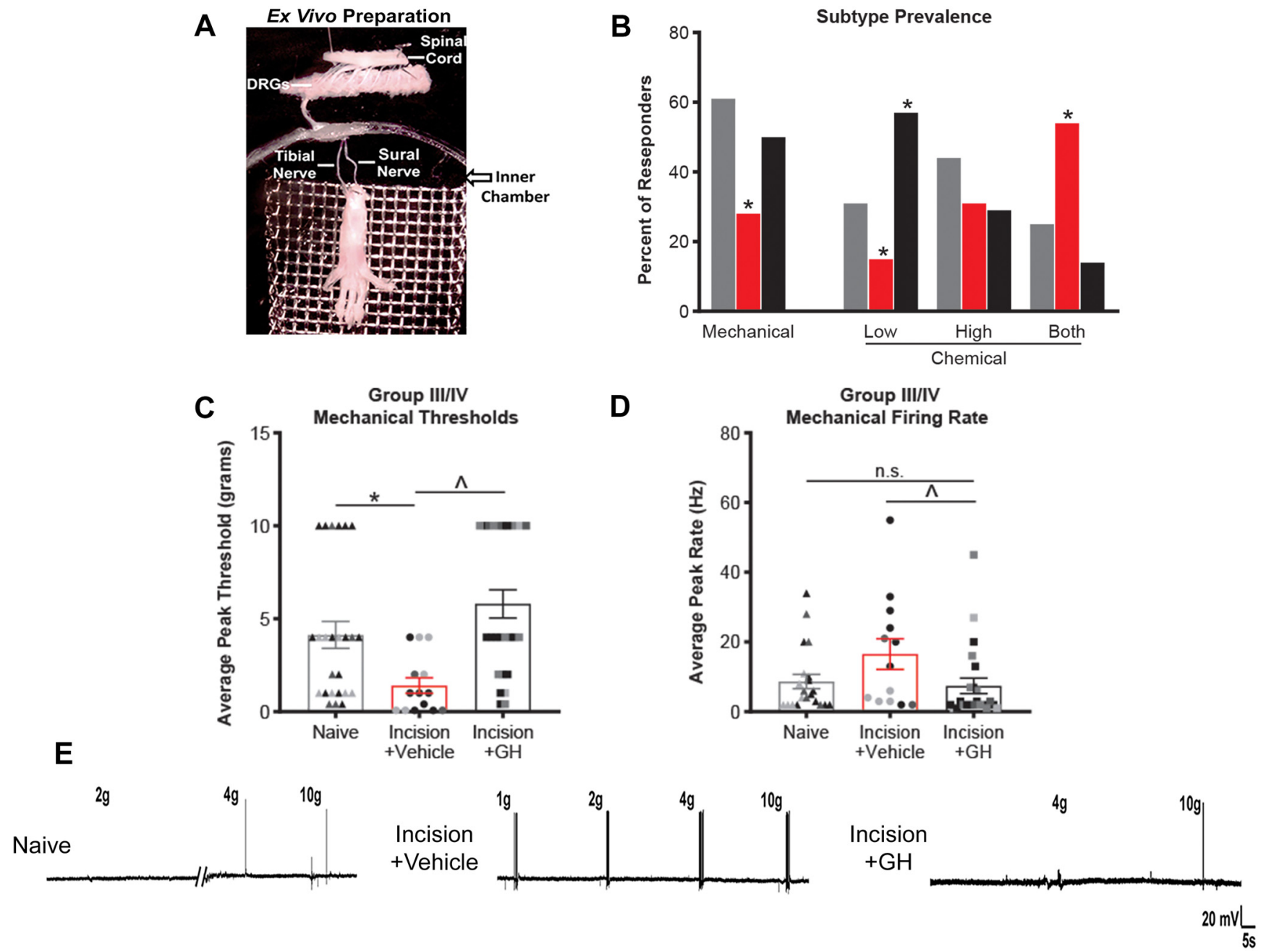

Figure 4. Intramuscular $\mathrm{GH}$ injection inhibits peripheral sensitization in neonates with incision. $\boldsymbol{A}$, Representative image of the hind paw muscle ex vivo recording preparation. $\boldsymbol{B}$, Prevalence of mechanically (naive vs veh.; $\chi^{2}=20.73,1 \mathrm{df}, * p<0.001$; naive vs $\mathrm{GH} \chi^{2}=2.02,1 \mathrm{df}, p=0.155$ ) and chemically [low (vs veh.; $\chi^{2}=6.35,1 \mathrm{df}, * p=0.012 ;$ vs $\mathrm{GH} \chi^{2}=12.68,1 \mathrm{df}$, $* p<0.001$ ), high (vs veh. and GH. $\chi^{2}=5.86,2 \mathrm{df}, p=0.053$ ), or both (vs veh.; $\chi^{2}=16.4,1 \mathrm{df}, * p<0.001$; vs GH $\chi^{2}=3.19,1 \mathrm{df}, p=0.074$ )] sensitive muscle afferents are altered by incision and rescued by GH treatment. C, von Frey threshold of mechanically sensitive Group III/IV primary afferents indicate overall effects $(H=14.79,2 \mathrm{df}, p<0.001)$. After Dunn's correction, we found that compared with naive animals ( $n=6$ animals, 51 cells), surgical injury results in sensitization in incised animals ( $* p=0.035$ vs naive, $n=8$ animals, 50 cells) that is blocked by GH treatment ( $p=0.441, n=10$ animals, 54 cells). Also, GH treatment altered thresholds compared with vehicle-injected incised animals $(\wedge p<0.001$ vs controls.) The shades of the data points indicate the age range of the animals (also true for $\mathrm{d}$ ). Light gray $=\leq P 7$. Medium gray $=P 8$ and P9. Dark gray $=P 10, P 11$. Black $=\geq P 12$. D, Mechanical FR showed similar results with an overall effect of $H=6.59,2 \mathrm{df}, p=0.037$. Adjusted $p$ value with Dunn's correction indicates that the average mechanical FR is decreased in injured animals treated with $\mathrm{GH}$ compared with those that were not treated after injury ( $\wedge p=0.037$ vs controls). ANOVA on ranks, Dunn's post hoc test. $\boldsymbol{E}$, Representative mechanical responses for each group are provided. Further analyses are conducted in Table 2. Data shown as mean \pm SEM. n.s., not significant.

significantly upregulated monocyte chemoattractant protein 1 (MCP-1), glial cell line-derived neurotrophic factor (GDNF), interleukin $1 \beta$ (IL1 $\beta$ ), and nerve growth factor (NGF), but not tumor necrosis factor $\alpha$ (TNF $\alpha$ ). Interestingly, none of these factors were altered by local GH treatment (Table 3). Together, data suggest that $\mathrm{GH}$ provides a tonic control of gene expression in the DRG, but exogenous GH treatment at low doses may not alter the inflammatory response to incision within the muscles.

\section{SRF upregulation in the DRGs modulates pain-related} behaviors following surgical incision

To begin to understand the mechanism by which GHr signaling effected neonatal nociception, we screened a number of known downstream transcription factors in the DRGs (Table 3). As we only see significant pain like behaviors and effects of local GH injection $1 \mathrm{~d}$ following incision, subsequent experiments evaluated this time point specifically. Neonates with muscle incision plus vehicle injection displayed significant upregulation of SRF, STAT3, and STAT5, and a significant downregulation of ELK3, but no changes in ELK 1 or ELK4. Injection of GH into the muscles after incision specifically blocked the injury-related upregulation of SRF and STAT5. Since genetic knock out of GHr in uninjured sensory neurons regulated SRF expression (above) and to determine the role that one of these factors may play in muscle incision-induced hypersensitivity in neonates, we used our in vivo nerve-targeted siRNA knock-down strategy to inhibit the DRG upregulation of SRF (Jankowski et al., 2009; Liu et al., 2017). Before incision, animals were injected with Penetratinlinked siRNAs against SRF (PenSRF) into the right sciatic nerve. We found that this strategy partially, but significantly blunted the upregulation of SRF in the DRGs at the mRNA and protein levels (Fig. $5 A, B$ ). We then assessed incision-related hypersensitivity in animals with the targeted knock down of SRF and found that inhibition of this transcription factor significantly reduced 
Table 2. Group III/IV IFs are unaffected between conditions within afferent subtypes

\begin{tabular}{|c|c|c|c|c|c|c|c|c|c|}
\hline \multirow{2}{*}{$\begin{array}{l}\text { Afferent type } \\
(\mathrm{Hz})\end{array}$} & \multicolumn{3}{|l|}{ Naïve } & \multicolumn{3}{|c|}{ Incision + vehicle } & \multicolumn{3}{|c|}{ Incision + GH } \\
\hline & Mean & SEM & $n$ & Mean & SEM & $n$ & Mean & SEM & $n$ \\
\hline \multicolumn{10}{|l|}{ Group III/IV IFs } \\
\hline Cold & 18.8 & 10.4 & 9.0 & 44.1 & 13.6 & 10.0 & 29.8 & 9.3 & 10.0 \\
\hline Hot & 59.1 & 1.8 & 2.0 & 51.3 & 21.6 & 3.0 & 78.2 & 39.7 & 8.0 \\
\hline Low metabolite & 24.7 & 22.3 & 4.0 & 4.5 & $\mathrm{n} / \mathrm{a}$ & 1.0 & 192.9 & 104.7 & 3.0 \\
\hline \multicolumn{10}{|c|}{ Group III/IV thermal and chemical FRs } \\
\hline Cold & 2.9 & 0.6 & 8.0 & 4.8 & 1.4 & 10.0 & 5.3 & 1.4 & 9.0 \\
\hline Hot & 16.0 & $\mathrm{n} / \mathrm{a}$ & 1.0 & 6.0 & 2.0 & 3.0 & 6.8 & 3.8 & 8.0 \\
\hline Low metabolite & 2.4 & 0.9 & 7.0 & 2.1 & 0.5 & 7.0 & 2.8 & 1.0 & 5.0 \\
\hline High metabolite & 3.4 & 0.1 & 7.0 & 5.8 & 3.0 & 9.0 & 1.0 & $\mathrm{n} / \mathrm{a}$ & 1.0 \\
\hline Both metabolite & 5.3 & 1.8 & 3.0 & 3.0 & 0.4 & 7.0 & 1.0 & $\mathrm{n} / \mathrm{a}$ & 1.0 \\
\hline
\end{tabular}

One-way ANOVA with Tukey's test or ANOVA on ranks with Dunn's post hoc test.

spontaneous paw guarding compared with neonates with control siRNA injection (PenCON) plus incision (Fig. 5C). Additionally, incision-related muscle mechanical hypersensitivity observed in mice with PenCON injection was also inhibited in mice with SRF knock-down (Fig. 5D). Interestingly, while PenSRF inhibited guarding in males, female guarding was unaffected by PenSRF injection (Fig. 5E). When analyzing mechanical hypersensitivity by sex, we found that PenSRF inhibited male mechanical hypersensitivity to incision; however, we were not able to detect mechanical hypersensitivity in the PenCON-injected females, as such effects of SRF knock-down on female mechanical responsiveness are inconclusive (Fig. 5F). Nevertheless, age distribution also showed no obvious effects in either group (embedded in Fig. 5C,D). These behavioral changes corresponded with inhibition of TRPA1 upregulation after SRF knock-down $(H=10.46$, df $=2, p=0.005)$ in the DRGs (PenCON/Veh+ Inc. $=307 \pm 65 \%, * p=0.007$, PenSRF + Inc. $=52 \pm 37 \%$, $p>0.05$; vs naive, $n=8-19$ /group). Interestingly, TRPV1 upregulation was not altered $\left[\left(F_{(2,18)}=15.53, p<0.001\right)\right.$, Tukey's correction: PenCON $=90 \pm 16 \%, * p=0.001$; PenSRF $=128 \pm 13 \%$, $* p<0.001, n=6-8$ /group]. Together, these data indicate one neuronal transcriptional pathway downstream of GHr that may modulate neonatal nociception.

\section{GH sequestering by infiltrating macrophages regulates} incision-related hypersensitivity in neonates

In our current and previous reports (Liu et al., 2017), we have shown that the levels of GH decrease in the injured tissue within $1 \mathrm{~d}$. However, the mechanism behind this reduction in GH levels were previously unknown. Using immunohistochemical analyses, we observed that GH is normally found in a diffuse pattern between myofibers within the muscles of naive neonates (Fig. $6 A)$. However, after incision, we found that $\mathrm{GH}$ was displaced in the muscle tissue into a more concentrated, localized manner (Fig. $6 B$ ). The areas of intense GH staining appeared to be monocyte-like based on qualitative morphologic assessments. We therefore used a transgenic mouse line in which a tdTomato reporter was expressed in monocytes and mature macrophages (LysM-Cre;td-Tom) and performed IHC for GH in these mice with incision. The concentrated pattern of GH staining in mice with incision was found to overlap significantly with the tdTomato reporter (Fig. 6C,D), while this is not observed in uninjured tissue which have few detectable macrophages (Fig. 6E).
Table 3. Transcriptional changes following a neonatal muscle incision and local GH injection are modulated in L3/4/5 DRGs but not in the injured muscle

\begin{tabular}{lcc}
\hline & Incision + & Incision + \\
Gene & Vehicle & GH \\
\hline \% Change in DRG mRNA (+/- = variance) & & \\
ASIC3 & $18 \pm 10 \%$ & $35 \pm 11 \%$ \\
ELK1 & $-29 \pm 13 \%$ & $-21 \pm 15 \%$ \\
ELK3 & $-97 \pm 5 \% *$ & $-98 \pm 15 \% *$ \\
ELK4 & $-28 \pm 8 \%$ & $-19 \pm 9 \%$ \\
Fcer2a & $-81 \pm 20 \% *$ & $-80 \pm 11 \% *$ \\
GHr & $15 \pm 4 \%$ & $16 \pm 6 \%$ \\
IGFr1 & $258 \pm 29 \% *$ & $21 \pm 14 \%$ \\
IL1-r & $-50 \pm 15 \% *$ & $-51 \pm 14 \% *$ \\
NF $\kappa$ B & $83 \pm 13 \% *$ & $40 \pm 11 \%$ \\
OSMr & $-59 \pm 17 \% *$ & $-66 \pm 16 \% *$ \\
P2X3 & $44 \pm 22 \% *$ & $-2 \pm 18 \%$ \\
P2Y1 & $44 \pm 15 \%$ & $-14 \pm 10 \%$ \\
SRF & $142 \pm 19 \% *$ & $27 \pm 25 \%$ \\
STAT1 & $92 \pm 28 \%$ & $10 \pm 18 \%$ \\
STAT3 & $220 \pm 7 \% *$ & $164 \pm 7 \% *$ \\
STAT5 & $164 \pm 20 \% *$ & $69 \pm 31 \%$ \\
TNFa-r & $15 \pm 8 \%$ & $13 \pm 7 \%$ \\
TRPA1 & $493 \pm 41 \% *$ & $1 \pm 25 \%$ \\
TRPV1 & $178 \pm 14 \% *$ & $12 \pm 15 \%$ \\
\% Change in muscle mRNA & & \\
GDNF & & $179 \pm 23 \% *$ \\
IL1 $\beta$ & $204 \pm 44 \% *$ & $608 \pm 40 \% *$ \\
MCP1 & $1098 \pm 50 \% *$ & $276 \pm 17 \% *$ \\
NGF & $584 \pm 24 \% *$ & $72 \pm 20 \% *$ \\
TNF $\alpha$ & $77 \pm 23 \% *$ & $-40 \pm 27 \%$ \\
\hline Da & $11 \pm 20 \%$ & \\
\hline
\end{tabular}

Data shown as a percent change from controls where the mean and SE of the difference of means are first calculated and then converted to percentages (minimum overall effect $F_{(2,8)}=4.517, p=0.049$ ), $* p<0.05$ versus controls; $n=3-12 /$ group, one-way ANOVA with Tukey's test or ANOVA on ranks, with Dunn's post hoc test. $n=1$ outlier $>2$ SDs away from the mean, ELK3 control data. Primer sequences are found in Table 1.

Previous work has suggested that macrophages can bind and internalize GH (Strous et al., 1996; Govers et al., 1999; Lu et al., 2013). Since tissue collection for our Western blot analyses was obtained from animals with cardiac perfusion and samples for IHC were obtained from snap-frozen, fresh tissues (un-perfused), we posited that the "reduction" in muscle GH levels observed after incision were because of macrophage dependent sequestering. We found that following cardiac perfusion with ice-cold saline in mice with incision, macrophages were no longer detected within the muscle (Fig. 6F). Moreover, in un-perfused neonates, there was no detectable reduction in $\mathrm{GH}$ using Western blot analysis (Fig. 6G). 
A

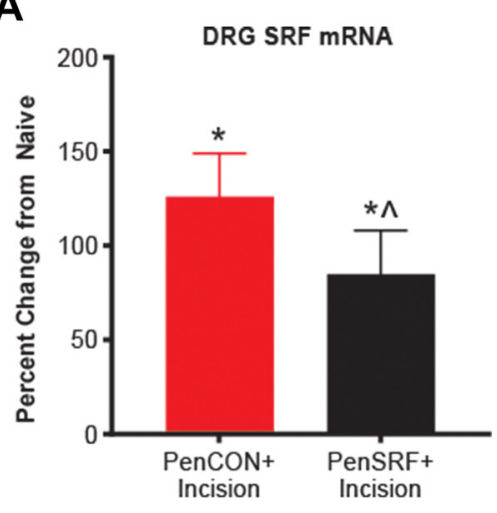

C

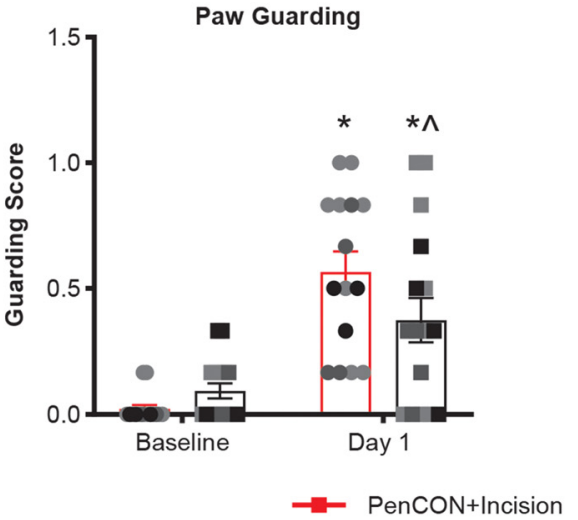

B

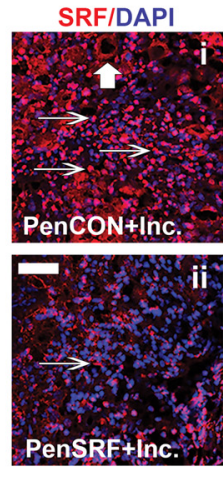

B'

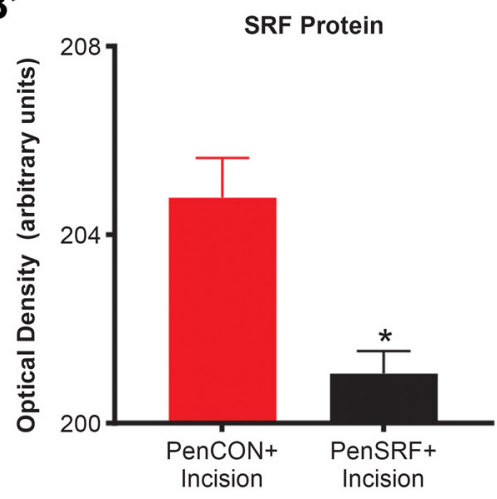

D

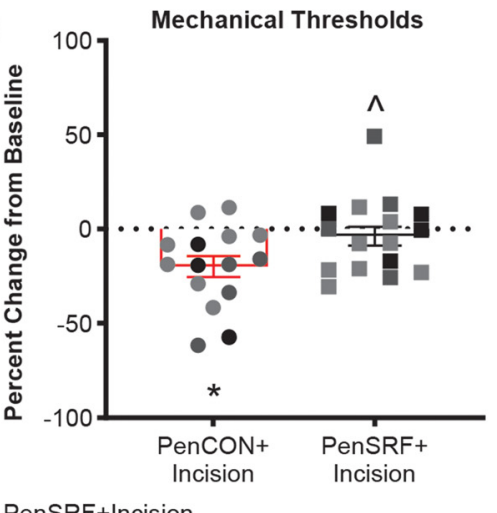

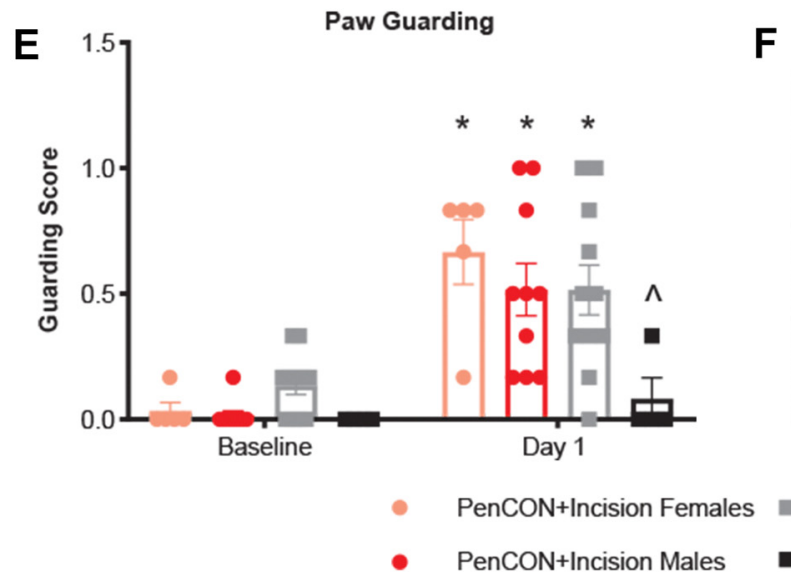

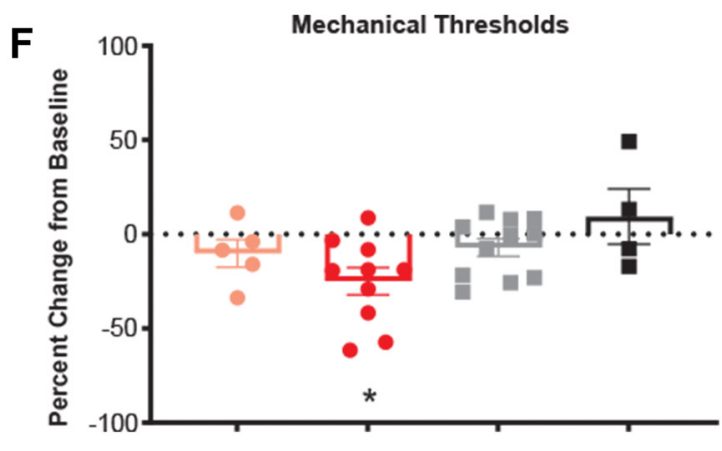

PenSRF+Incision Females

PenSRF+Incision Males

Figure 5. Nerve-targeted knock down of SRF upregulation blunts pain-like responses after muscle incision. $\boldsymbol{A}$, Injection of SRF targeting siRNAs (PenSRF) into the sciatic nerve alters SRF mRNA $\left(F_{(2,45)}=17.16, p<0.001\right)$. Adjusted $p$ values indicate that both groups increase mRNA after injury ( $* p<0.001$ vs naive) and that DRGs injected with PenSRF have significantly lower levels than controls [ $p=0.025$ vs controls (PenCON)]; $n=7$ naive, 22 PenCON, 19 PenSRF, one-way ANOVA, Holm-Sidak post hoc test. $n=1$ outlier $>2$ SDs away from the mean, knock down data. $\boldsymbol{B}$, Representative images (Bi, Bii) display similar results at the protein level, arrows indicate SRF+ staining, big arrow indicates satellite cell, $\left(\boldsymbol{B}^{\prime}\right)$ using immunocytochemical labeling and mean staining value of a ROI (red). DAPI (blue) c0-stain was used to mark all nuclei. $F_{(1,4)}=14.71, * p<0.05$ versus PenCON; $n=3$ animals/group, Tukey's post hoc test. $C$, In behavioral guarding experiments we detected an overall effect of day $\left(F_{(1,29)}=58.39, p<0.001\right)$ and an interaction $\left(F_{(1,29)}=5.93, p=0.021\right)$. Tukey's correction indicated that incised animals show increased paw guarding $(* p<0.001$ vs $\mathrm{BL})$, and animals injected with PenSRF showed reduced guarding compared with the PenCON-injected mice ( ${ }^{\wedge} p=0.035$ vs controls). The shades of the data points indicate the age range of the animal at BL behavior (also true for d). Light gray $=\leq P$. Medium gray $=P 8$ and P9. Dark gray $=P 10, P 11$. Black $=\geq P 12$. D, For mechanical withdrawal behavior a main effect of day $\left(F_{(1,29)}=9.09, p=0.005\right)$ and an interaction $\left(F_{(1,29)}=4.97, p=0.034\right)$ was detected. PenCON-incised animals have reduced mechanical withdrawal thresholds compared with BL $(* p=0.001$ vs BL) while PenSRF-injected animals do not $(p=0.577)$. Further, a difference between the groups was detected within day 1 ( $\wedge=0.042$ vs controls); $n=15-16 /$ group, two-way RM ANOVA, with Tukey's post hoc test. Data shown as mean \pm SEM or percent change from naive. $\boldsymbol{E}, \boldsymbol{F}$, We also detected an overall effect of sex $\left(F_{(1,27)}=8.26, p=0.0078\right)$ in our guarding assay but not in our muscle squeezing assay $\left(F_{(1,27)}=0.49\right.$, $p=0.489$ ). In guarding, we found that females injected with PenSRF also have significantly increased guarding at $1 \mathrm{~d}(* p=0.035$ vs BL). Males injected with PenSRF do not differ from BL ( $* p=0.999$ vs $\mathrm{BL}$ ) and guard significantly less than females ( $(\hat{p}=0.01$ vs PenSRF female within day 1 ). When sex is delineated in muscle squeezing behaviors, we find that only males injected with PenCON display a significant change from BL $(* p=0.0135$ vs BL). PenCON males $=10$, females $=5$; PenSRF males $=5$, females $=11$. Three-way RM ANOVA (GraphPad v.8; time $\times$ sex $\times$ condition), Tukey's post hoc test. Data shown as percent change from naive or mean \pm SEM.

Data thus suggested that under neonatal injury conditions, infiltrating macrophages may sequester $\mathrm{GH}$ and thereby effectively remove the tonic signal that $\mathrm{GH}$ normally provides to innervating primary afferents. To test this hypothesis, we first treated incised animals intramuscularly with clodronate liposomes to deplete the infiltrating macrophages. Following surgery, this depletion prevented evoked muscle hypersensitivity but not spontaneous paw guarding behaviors (Fig. 7A,B). As 

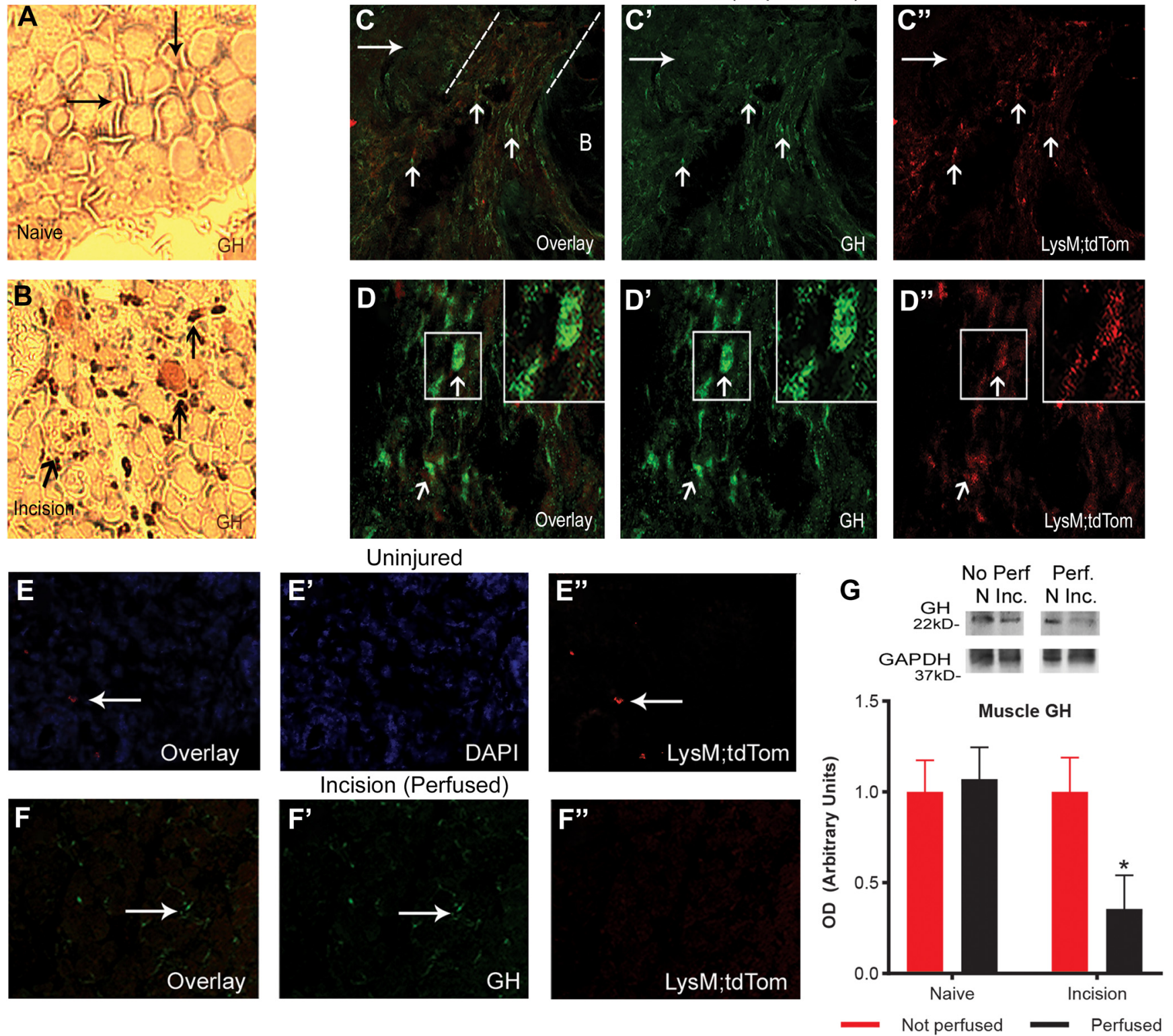

Figure 6. Muscle GH distribution is altered by injury and macrophage presence is diminished following cardiac perfusion. $\boldsymbol{A}$, Immunostaining for GH (arrows) in a cross section of hindpaw muscle of a naive animal with DAB staining. $\boldsymbol{B}$, GH staining (arrows) in the muscle of an animal $1 \mathrm{~d}$ after an incision. $\boldsymbol{C}$, Overlay of macrophages and GH (arrows) in the muscles of a LysMcre/ tdTomato reporter animal using an antibody stain for GH (green; (') and tdTom reporter for macrophages (red; (') $1 \mathrm{~d}$ after an injury of the hindpaw muscle. $\boldsymbol{D}$ - $\boldsymbol{D}^{\prime \prime}$, Representative higher magnification images of macrophage and GH overlay (arrows) in injured muscle. $\boldsymbol{E}$, Representative images of uninjured hindpaw muscle from the macrophage reporter (LysM;tdTom) mouse co-stained with DAPI (blue) to mark nuclei. Arrows show few macrophages present in uninjured tissue. $\boldsymbol{F}$, Images of injured hindpaw muscle in perfused LysM;tdTom animals (red) and stained for GH (green). Arrows indicate some remaining GH staining. G, Representative Western blottings for GH in naive and injured muscle with and without cardiac perfusion. Quantification of Western blot analysis indicates a main effect of injury $\left(F_{(1,12)}=5.58, p=0.036\right)$ and an interaction $\left(F_{(1,12)}=7.32, p=0.019\right)$ for WT animals. We saw no main effects in MacGHr $-/-$ animals. Tukey's correction explained that only WT animals that are incised and perfused have reduced levels of muscle GH compared with naive $(* p=0.004$ vs controls); $n=4 /$ group, two-way ANOVA, Tukey's post hoc test. Data shown as the mean \pm SEM.

macrophages appeared to at least play some role in incisionrelated hypersensitivity in neonates, we then analyzed incised animals with the GHr knocked out in macrophages and mature monocytes (LysM-Cre; $\mathrm{GHr}^{\mathrm{f} / \mathrm{f}} ; \mathrm{MacGHr}-/-$ ). Our injury experiments up to this point were completed in Swiss Webster male and female animals. As the MacGHr-/- mice were bred on a C57Bl/6 background, we compared the two strains in both males and females and found no difference in pain-like behaviors between strains (Fig. 7C,D).

Following this, we then found that animals with the GHr knocked out in macrophages still display infiltration of these immune cells within the muscles after incision, but they do not contain GH (Fig. 8A). We also found no reduction in muscle $\mathrm{GH}$ levels in these mice after injury and perfusion, unlike that observed in WT animals (Fig. 8B). The MacGHr-/- mice also displayed significantly less paw guarding (Fig. 8C) and did not exhibit reduced muscle withdrawal thresholds $1 \mathrm{~d}$ after injury (Fig. 8D) unlike control mice. Mechanical hypersensitivity from the injury was not detected at $3 \mathrm{~d}$ in either group, but unlike MacGHr-/- mice, controls still displayed a small guarding response at $3 \mathrm{~d}$ (Fig. $8 E, F)$. Similar to other conditions, no effect of age within the 
P7-P14 time period was detected under these conditions (embedded in Fig. 7C,D).

We also analyzed the transcriptional changes within DRGs and the injured muscles from these mice. Receptors and channels shown previously (Table 3 ) to be upregulated in animals after injury were also observed in control mice with incision, but these same receptors were not upregulated in MacGHr-/- animals with incision (Fig. 8G). However, the upregulation of muscle cytokines and growth factors observed in control animals with incision were unaffected by knock-out of GHr in macrophages. In fact, the levels of some of these factors were significantly greater in MacGHr-/- mice with incision compared with incised controls (Fig. 8H). These data indicate that macrophages may sequester muscle GH after injury, likely removing the tonic inhibition that $\mathrm{GH}$ provides on primary afferent neurons and leads to injuryinduced pain-related behaviors.

\section{Early life GH reduction alters neonatal} "priming" of nociceptive responses to injury later in life

To determine whether GH-related anti-nociception could modulate the prolonged effects of repeated injury, we first assessed whether $\mathrm{GH}$ deficiency alone could induce a prolonged hypersensitivity after incision. In order to test this, we used the GHRHr knock-out mice that we have previously shown to display neonatalspecific hypersensitivity (Ford et al., 2019) and performed a single incision in these mice at P35. At BL, we detected no difference between WT controls (GHRHr+/+), heterozygous GHRHr mutants (GHRHr+/-) and the homozygous GHRHr knock-out (GHRHr-/-) mice. However, GHRHr-/ - animals and to a degree, $\mathrm{GHRHr}+$ / - mice, displayed prolonged spontaneous paw guarding (Fig. 9A) and muscle withdrawal thresholds (Fig. 9B) after P35 incision compared with WT C57Bl/6 controls. In Swiss Webster animals, we then confirmed previous reports (Walker et al., 2009; Moriarty et al., 2018) that an early life muscle incision (P7) in WT control mice followed by a P35 incision resulted in a longer lasting mechanical hypersensitivity compared with mice with P35 incision alone. Interestingly, if treatment with GH was given at the time of the early life injury (P7), prolonged hypersensitivity was not detected after the second incision (Fig. 9C). These data support our earlier findings that $\mathrm{GHr}$ signaling in early life is important for sensitization and disruptions in this signaling can influence long-term somatosensory alterations in response to injury later in life.

\section{Discussion}

Our data indicate that in neonates, GH expressed throughout the hindpaw muscles tonically activates the GHr on neurons. This maintains basal transcription factor levels in sensory neurons, which supports normal afferent function. However, after an early life injury, macrophage infiltration results in an immune cell-dependent sequestration of muscle GH. The displacement of GH within the muscle removes $\mathrm{GH}$ signaling to neurons resulting, at least in part, in SRF-dependent transcription of sensory-related receptors/channels in the affected DRGs. Restoring the basal levels of GH (through targeted injections or by preventing the macrophage dependent sequestration), maintains neuronal GHr signaling and blocks peripheral sensitization (Fig. 10). GH signaling to immature neurons also appears to modulate injury responses later in life (Fig. 10). Thus, in neonates, a unique immune system dependent modulation of endocrine communication with the peripheral nervous system is accessed after injury to regulate nociception.

Nociception involves a variety of distinct extracellular and intracellular neuronal mechanisms to transduce sensory stimuli into the CNS (Julius and Basbaum, 2001). Throughout development, distinct neurotrophic factors regulate neuronal subtype formation and innervation, with recent work describing non-canonical roles (Shelton and Reichardt, 1984; Mendell, 1996; Moqrich et al., 2004). Additionally, developing sensory neurons display changing 
A

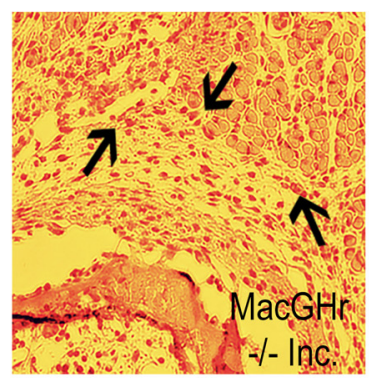

B

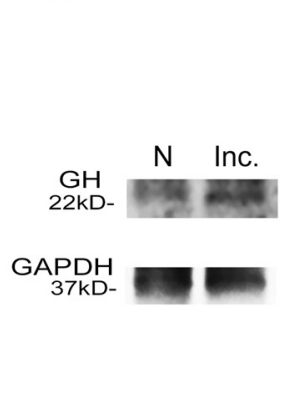

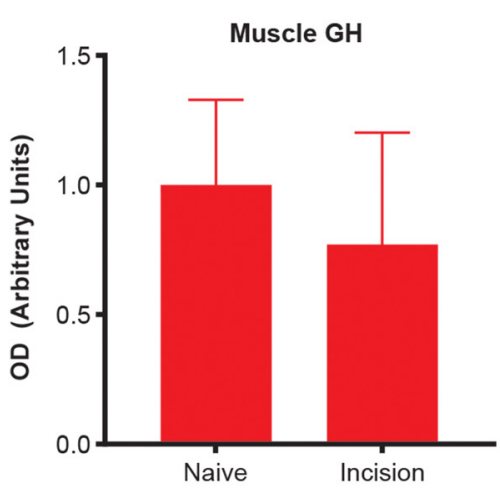

E

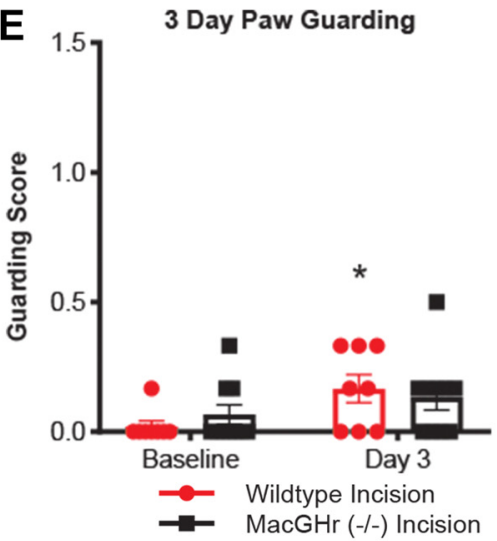

C

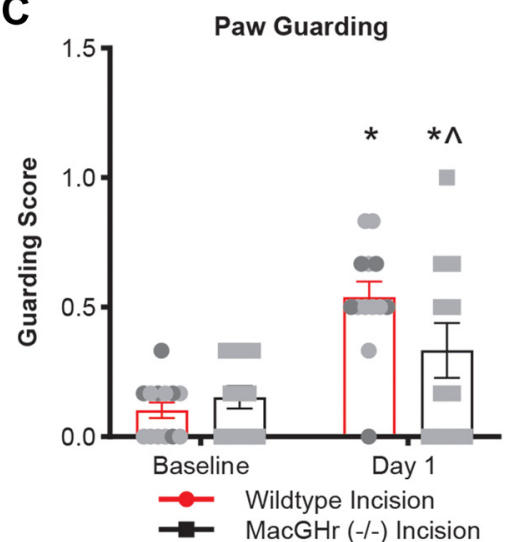

$\mathbf{F}$

F 3 Day Mechanical Thresholds

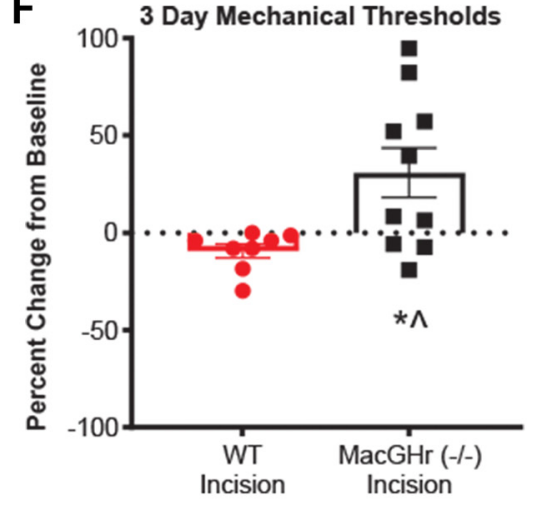

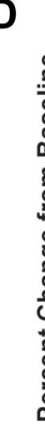

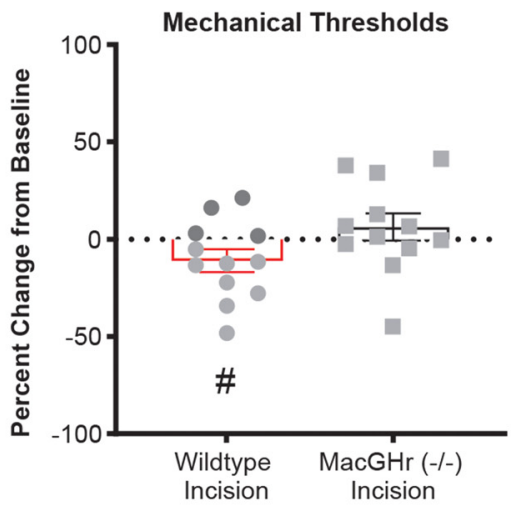

\begin{tabular}{|ccc|}
\hline \multirow{3}{*}{$\%$ Change in Muscle mRNA } \\
\cline { 2 - 3 } Gene & Incised & Incised \\
MCP1 & $664 \pm 33 \% *$ & $\frac{\text { MacGHr }(-/ \cdot)}{1902 \pm 31 \%^{*}}$ \\
IL1 $\beta$ & $186 \pm 57 \%$ & $8327 \pm 32 \%^{\star \wedge}$ \\
NGF & $-7 \pm 20 \%$ & $275 \pm 6 \%^{* \Lambda}$ \\
TNFa & $189 \pm 36 \% \%^{*}$ & $655 \pm 9 \%^{* \Lambda}$ \\
\hline
\end{tabular}

Figure 8. GHr signaling on macrophages is necessary for incision related pain-like behaviors and DRG molecular sensitization. $\boldsymbol{A}$, Representative image of $\mathrm{GH}$ staining (dashed arrow) using DAB in the muscles of an injured macrophage-specific GHr knock-out ( $\mathrm{MacGHr}-/-$ ) animal. Hematoxylin (light red) dense cells show infiltrating macrophages (solid arrows). $\boldsymbol{B}$, Representative Western blottings and quantification of GH levels after injury in MacGHr- - - animals. $C$, MacGHr knock-out animals display main effects of day $\left(F_{(1,22)}=32.19, p<0.001\right)$ and an interaction $\left(F_{(1,22)}=4.59, p=0.044\right)$ for guarding. Tukey's correction indicates that both WT ( $* p<0.001$ vs BL) and MachGHr $-/-(* p=0.025$ vs BL) guard at $1 \mathrm{~d}$, but MacGHr $-/-$ animals guard less than controls ( $\wedge p=0.047$ vs controls) $n=1$ outlier $>2$ SDs away from the mean, control group. The shades of the data points indicate the age range of the animal at BL behavior (also true in $\boldsymbol{D}$ ). Light gray $=\leq P$. Medium gray $=P 8$ and P9. Dark gray $=P 10$, P11. Black $=\geq P 12$. D, In muscle squeezing assays, we found MacGHr $-/-$ mice do not show reduced muscle withdrawal thresholds after injury $\left(F_{(1,22)}=1.27, p=0.272\right)$ and when compared with knock-out animals as a percent change, WT animals demonstrate a mild change $\left(F_{(1,22)}=3.64\right.$, $\# p=0.07) ; n=12-13$ /group, one-way or two-way ANOVA, with Tukey's post hoc test. $\boldsymbol{E}$, Three days after a neonatal incision, we see only an effect of day $\left(F_{(1,16)}=5.99, p=0.026\right)$. Tukey's correction indicated that $\mathrm{MaCGHr}-1-$ animals no longer display a difference from BL $(p=0.266)$, but $(57$ animals still slightly guard their paw $(* p=0.039 \mathrm{vs} \mathrm{BL})$. $F$, In muscle squeezing assays, we detected a main effect of genotype $\left(F_{(1,16)}=30.66, p<0.001\right)$ and an interaction $\left(F_{(1,16)}=8.44, p=0.01\right)$. Mechanical withdrawal thresholds are increased in MacGHr $-/-3 \mathrm{~d}$ after a neonatal injury compared with their BL ( $* p=0.017$ vs BL) and controls ( $~ p p=0.043$ vs controls); $n=8-10 /$ group, two-way RM ANOVA, Tukey's post hoc test. G, Upregulation of select receptors/channels in the DRGs are observed in WT control mice with incision, but this is not found in incised MacGHr-/-DRGs. $\boldsymbol{H}$, Incision-induced upregulation of select cytokines and growth factors in the muscles is found in both control and MacGHr-/- mice; $* p<0.05$ versus naive, $\hat{p}<0.05$ versus WT incised; $n=3-4 /$ group, two-way ANOVA, Tukey's post hoc test. Data shown as mean \pm SEM or percent change from naive.

transcriptional identities and functional maturation from the neonatal period through adulthood (Jankowski et al., 2014; Adelman et al., 2019; Sharma et al., 2020). While growth factors clearly alter subtype survival, we detect that modulation of neuronal GH signaling results in functional changes to normal somatosensory processing in both the skin (Ford et al., 2019) and the muscle. In our sensory neuron-specific deletion of $\mathrm{GHr}$, cutaneous behaviors are robustly altered in addition to muscle driven behaviors. This corroborates our previous data that focused on $\mathrm{GH}$ signaling in cutaneous afferents (Liu et al., 2017; Ford et al., 2019). Here, we were able to describe a mechanism of sensitization that occurs in muscle afferents. GH may have a more global effect on afferent function that influences somatosensory development unique to that observed with neurotrophic factors.

The developmental stage of the animal may affect how primary afferents respond to injury (Jankowski et al., 2014). Our previous and current reports indicate that muscle afferent sensitization occurs similarly across the lifespan but may become insensitive to $\mathrm{GH}$ as an antinociceptive treatment beginning in adolescence (Figs. 2, 4; Queme et al., 2020). GH may have agedependent and specific roles in regulating multiple cell types in 
A

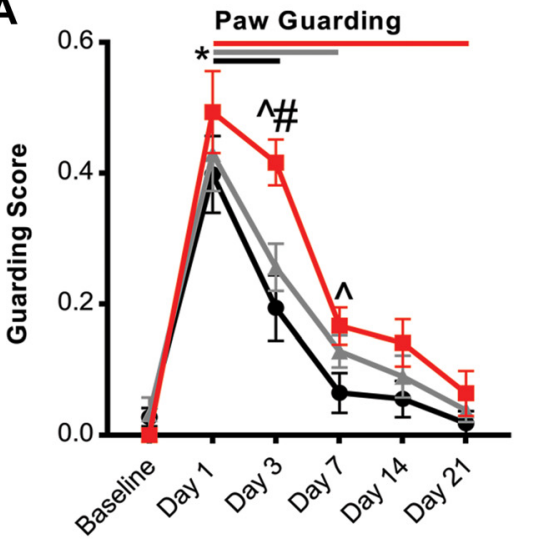

B

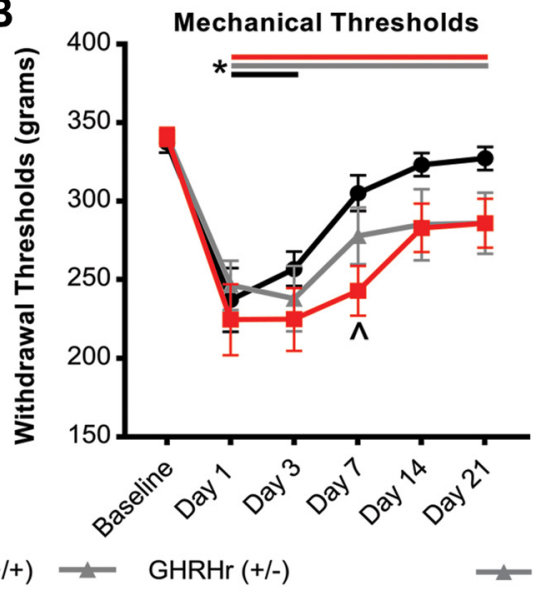

C

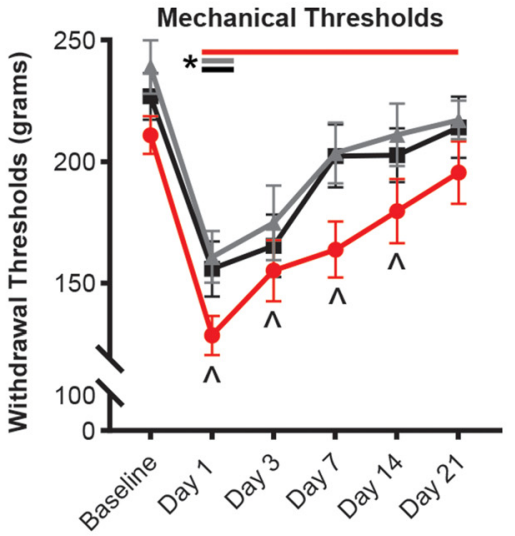

P35 Incision Only $\longrightarrow$ P7 Incision + P35 Incision

$\rightarrow$ GHRHr $(-/-)$

Figure 9. Alterations in GH levels during early postnatal development modulate the behavioral responses to incision in adolescence. $A$, We found that $\mathrm{GHRH}-/-$ animals injured later in life demonstrate main effects of day through day 14 , effect of genotype on day $3\left(F_{(2,32)}=3.76, p=0.034\right)$, and an interaction on day $3\left(F_{(2,32)}=14.18, p<0.001\right)$ and day $7\left(F_{(2,32)}=4.08\right.$, $p=0.026)$. Tukey's correction explained that WT (C57/BI6) animals guard through day 3 after an injury, $\mathrm{GHRH}+/-$ animals guard through day 7 after injury and $\mathrm{GHRHr}-/-$ animals guard through day 21 ( $* p<0.05$ vs $\mathrm{BL}$ ). We also detect that GHRHr $-/$ - animals guard more than GHRHr $+/-(\# p<0.001)$ and WT $3 \mathrm{~d}$ after the injury, an effect that continues through day 7 when compared with WT animals ( $\wedge p 0.05$ vs WT). $B$, Muscle mechanical hypersensitivity is also more severe and prolonged in GHRHr $+/-$ and GHRHr $-/-$ mice, displaying a main effect of day through day 21 and an interaction effect at day $7\left(F_{(2,32)}=3.81, p=0.033\right)$. WT animals only displayed mechanical hypersensitivity through day 3 , while $\mathrm{GHRHr}+/-$ and $\mathrm{GHRHr}+/+$ are hypersensitive through day 21 (*p $<0.01$ vs BL). Also, at day $7, \mathrm{GHRHr}-/-$ animals withdrew at significantly lower thresholds compared with WT animals ( ${ }^{\wedge} p=0.003$ vs controls); $*$ is indicated for each group by the colored horizontal bars, the ending of the bar indicates the ending of the detected significance; $n=9-13 /$ group, two-way RM ANOVA with Tukey's test or ANOVA on ranks with Dunn's post hoc test. C, Muscle withdrawal thresholds in WT Swiss Webster mice following a P7 plus P35 incision are prolonged compared with mice that only received a single injury at $\mathrm{P} 35$. We detect an effect of day at all time points and a moderate effect of injection at day $7\left(F_{(2,32)}=2.86, p=0.072\right)$. This effect is blocked by an intra-peritoneal injection of GH during P7 incision; $* p<0.05$ versus BL (indicated as in $\boldsymbol{A}, \boldsymbol{B}$ ); ${ }^{\wedge} p<0.05$ versus time-matched P35 incision only; $n=11$-12/group, two-way RM ANOVA, with Tukey's post hoc test. Data shown as the mean \pm SEM.

the periphery after insult. We found that sensory neuron GHr is critical to prevent sensitization (Fig. 1) but we have not yet delineated the cell type-specific effects of this knock-out. It will be interesting to evaluate a nociceptor-specific knock out of GHr to possibly replicate these findings in the neurons hypothesized to be driving the response. Because of the unique developmental responses, neonatal injury may engage a distinct cellular interaction through which $\mathrm{GH}$ ultimately modulates neonatal nociception and injury-related hypersensitivity. It is worth noting that the ages we detect GH-related effects on nociception are also when systemic $\mathrm{GH}$ is at its highest, and is critical for overall growth and development (Müller et al., 1999; Fig. 2). Together, this evidence indicates a critical period in which the nervous, immune and endocrine systems uniquely interact to modulate peripheral sensitization.

Following a noxious stimulus, primary afferents respond by initiating various intracellular cascades that alter how the neuron responds to specific sensory stimuli (Basbaum et al., 2009). In our current work, we found that neonatal incision upregulated transcription factors SRF and STAT5 in the DRGs, which could be blocked by intramuscular GH treatment (Fig. 2; Table 3). However, only SRF was found to be altered in the DRGs in our sensory neuron GHr knockout animal (see Results). SRF operates in a complex to regulate gene expression in an age-controlled manner and is downstream of

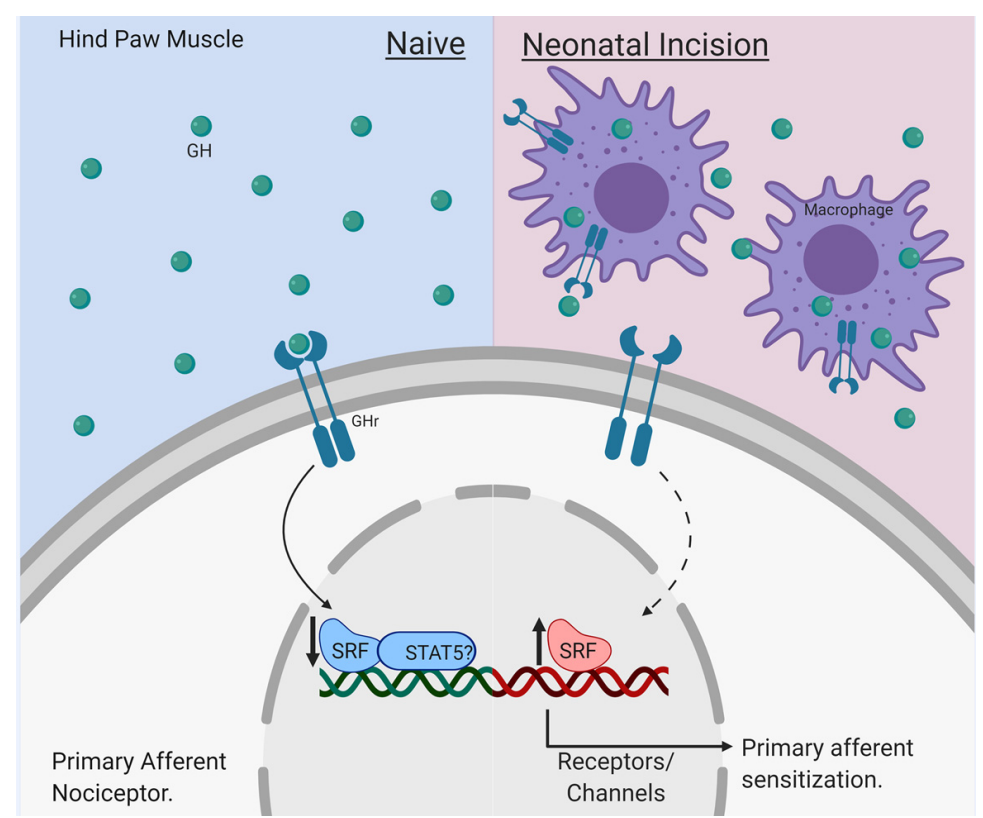

Figure 10. Role of GH in neonatal nociception. Under naive conditions (left), GH is diffusely available throughout the neonatal muscle tissue, allowing persistent activation of the GHr on primary afferent nociceptors. Normal activation of neuronal GHr maintains homeostatic transcription levels and regulates sensitizing factors such as TRPA1 and TRPV1 partially through SRF-dependent regulation. Following a neonatal injury (right), macrophages infiltrate the injured tissue and sequester GH, thereby effectively reducing GH availability to the nociceptors. The removal of tonic GH signaling to neurons permits SRF (and possibly STAT5) dependent transcription of various receptors and channels, leading to nociceptor sensitization and pain-related hypersensitivity after injury. Created with BioRender.com. 
canonical GH signaling (Zhang et al., 2014; Carter-Su et al., 2016). Although we did not directly target this factor in the $\mathrm{Adv} ; \mathrm{GHr}^{\mathrm{f} / \mathrm{f}}$ mice, we found that incision-induced upregulation of SRF is important for at least TRPA1, but not TRPV1 upregulation after incision. STAT5 is another interesting target that may have the ability to transcriptionally control TRPV1 (TargetScanMouse and miRDB), but is not upregulated because of $\mathrm{GHr}$ related signaling pathways (Kao et al., 2012; Gouin et al., 2017). It is likely that a number of alterations are induced by both injury and GH signaling resulting in an intracellular dynamic that collectively modulates sensitization. Further analyses will be necessary to determine this.

Immune system regulation of the affected microenvironment is a well-known phenomenon following many different injuries and has been noted to be distinct in neonates (Weston et al., 1977; Julius and Basbaum, 2001; Basbaum et al., 2009; Winterberg et al., 2015; Kumar and Bhat, 2016). The peripheral immune system reacts to an injury by mobilizing in stages with macrophages being one of the first responders (Fig. 6). This effect may be more heavily relied on in neonates that have yet to fully develop (Winterberg et al., 2015). We detected a time-dependent window for the local injection of GH that, if given $8 \mathrm{~h}$ after the injury, only partially blocks sensitization (Fig. $2 G$ ). It may be that this time point corresponds with the infiltration of macrophages to the injured area, and only at this time is free GH sequestered initiating the GH-dependent sensitization of nociceptors. When macrophages were eliminated by liposome clodronate, pain-like phenotypes may have been prevented in part because the tonic GH signal on sensory neurons is maintained (Fig. 7A,B). Previous reports indicate that the use of free $\mathrm{GH}$ by macrophages is necessary for proper control over their production of cytokines and growth factors which subsequently regulates the inflammatory response to injury ( $\mathrm{Lu}$ et al., 2013). We found that the prevention of GH signaling to macrophages by specific deletion of the $\mathrm{GHr}$ in these cells resulted in a reduced control over injury-site-specific cytokine levels (Fig. 8G,H; Lu et al., 2013; Kumar et al., 2014). Interestingly, recent work has indicated an opposite effect, demonstrating that treating macrophages in culture with $\mathrm{GH}$ also results in an increased proinflammatory cytokine release, suggesting that GH is a homeostatic regulator of macrophages (Dehkhoda et al., 2018; Schneider et al., 2019). Despite the increase of these factors known to be pro-nociceptive (Sommer and Kress, 2004), our manipulation, which rescued muscle GH levels, prevented sensitization and pain-like behaviors. We also note that the receptors of some cytokines, such as the IL1 $\beta$ receptor, are downregulated in DRG after a neonatal injury which may account for this discrepancy (Table 3). It may be that restoration of GH signaling in neurons supersedes pro-nociceptive cytokine signaling (Figs. 1, 2,8 ; Table 3). Regardless, when comparing our macrophage-specific GHr knock-out data with our sensory neuron-specific knock-out data, it is clear that a disruption in sensory neuron $\mathrm{GH}$-signaling strongly modulates neonatal nociception.

Early life injury induces neuronal and immunologic alterations that, when re-activated by an insult later in life, results in enhanced pain-like outcomes (Ren et al., 2004; Boissé et al., 2005; Walker et al., 2009; Schwaller et al., 2015; Zhong et al., 2018; Moriarty et al., 2019). While a number of analyses of the central nervous and immune systems have confirmed that this "priming" effect requires central processing, it is clear that primary afferent input is also necessary (Beggs et al., 2012; Walker et al., 2016; Moriarty et al., 2018, 2019). Our work suggests that GH prevents aspects of primary afferent sensitization (Fig. 4), and so we posited that GH may also influence the priming of neonatal injury. GH deficiency which induces a neonatal-specific hypersensitivity (Ford et al., 2019) is sufficient to prolong the normal behavioral responses to surgical insult or, as a recent study found, a formalin challenge (Fig. 9; Leone et al., 2019). These data suggest that GH levels and immune modulation are important for the induction of the early life injury priming effect and that this system can be manipulated by exogenous $\mathrm{GH}$ treatment.

Here, we describe a potentially neonatal-specific mechanism of nociception as well as an intervention that is effective at blocking pain-like behaviors and primary afferent sensitization. Correlations between the level of circulating GH levels and pain in patients has been previously observed in patients deficient in the hormone (Cimaz et al., 2001). Interestingly, patients that suffer from widespread muscle pain (fibromyalgia) sometimes also have altered GH levels and can be treated with GH for pain. These results may have important clinical implications as many children are not diagnosed with GH deficiency until they are a few years of age, in which time they may have already induced a priming effect to later in life injuries such as surgery. This is an important line of future research that will be necessary to evaluate in humans. Together with the current and previous work (Liu et al., 2017), data suggest that interventions designed to control the local levels of GH may be clinically beneficial for pain in young children.

\section{References}

Adelman P, Baumbauer K, Friedman R, Shah M, Wright M, Young E, Jankowski MP, Albers KM, Koerber HR (2019) Single cell q-PCR derived expression profiles of identified sensory neurons. bioRxiv. doi: 10.1101/ 560672.

Baccei ML (2016) Rewiring of developing spinal nociceptive circuits by neonatal injury and its implications for pediatric chronic pain. Children 3:16.

Baoge L, Van Den Steen E, Rimbaut S, Philips N, Witvrouw E, Almqvist KF, Vanderstraeten G, Vanden Bossche LC (2012) Treatment of skeletal muscle injury: a review. ISRN Orthop 2012:689012.

Bartholomew EM, Nath JL (2009) Fundamentals of anatomy and physiology, pp 616-617. Upper Saddle River: Pearson Education Inc.

Basbaum AI, Bautista DM, Scherrer G, Julius D (2009) Cellular and molecular mechanisms of pain. Cell 139:267-284.

Beggs S, Currie G, Salter MW, Fitzgerald M, Walker SM (2012) Priming of adult pain responses by neonatal pain experience: maintenance by central neuroimmune activity. Brain 135:404-417.

Boissé L, Spencer SJ, Mouihate A, Vergnolle N, Pittman QJ (2005) Neonatal immune challenge alters nociception in the adult rat. Pain 119:133-141.

Brennan TJ, Vandermeulen EP, Gebhart GF (1996) Characterization of a rat model of incisional pain. Pain 64:493-501.

Brewer CL, Baccei ML (2020) The development of pain circuits and unique effects of neonatal injury. J Neural Transm (Vienna) 127:467-479.

Brewer CL, Li J, O’Conor K, Serafin EK, Baccei ML (2020) Neonatal injury evokes persistent deficits in dynorphin inhibitory circuits within the adult mouse superficial dorsal horn. J Neurosci 40:3882-3895.

Brust V, Schindler PM, Lewejohann L (2015) Lifetime development of behavioural phenotype in the house mouse (Mus musculus). Front Zool 12:S17.

Carter-Su C, Schwartz J, Argetsinger LS (2016) Growth hormone signaling pathways. Growth Horm IGF Res 28:11-15.

Ceseña TI, Cui TX, Piwien-Pilipuk G, Kaplani J, Calinescu A-A, Huo JS, Iñiguez-Lluhí JA, Kwok R, Schwartz J (2007) Multiple mechanisms of growth hormone-regulated gene transcription. Mol Genet Metab 90:126133.

Cimaz R, Rusconi R, Fossali E, Careddu P (2001) Unexpected healing of cutaneous ulcers in a short child. Lancet 358:211-212.

Cuatrecasas G (2009) Fibromyalgic syndromes: could growth hormone therapy be beneficial? Suppl Pediatr Endocr Rev 6 [Suppl 4]:529-533.

Cuatrecasas G, Riudavets C, Güell MA, Nadal A (2007) Growth hormone as concomitant treatment in severe fibromyalgia associated with low IGF-1 serum levels. A pilot study. BMC Musculoskelet Disord 8:119. 
Cuatrecasas G, Gonzalez MJ, Alegre C, Sesmilo G, Fernandez-Solà J, Casanueva FF, Garcia-Fructuoso F, Poca-Dias V, Izquierdo JP, PuigDomingo M (2010) High prevalence of growth hormone deficiency in severe fibromyalgia syndromes. J Clin Endocrinol Metab 95:4331-4337.

Cuatrecasas G, Alegre C, Fernandez-Solà J, Gonzalez MJ, Garcia-Fructuoso F, Poca-Dias V, Nadal A, Cuatrecasas G, Navarro F, Mera A, Lage M, Peinó R, Casanueva F, Liñan C, Sesmilo G, Coves MJ, Izquierdo JP, Alvarez I, Granados E, Puig-Domingo M (2012) Growth hormone treatment for sustained pain reduction and improvement in quality of life in severe fibromyalgia. Pain 153:1382-1389.

Cuatrecasas G, Alegre C, Casanueva FF (2014) GH/IGF1 axis disturbances in the fibromyalgia syndrome: is there a rationale for $\mathrm{GH}$ treatment? Pituitary 17:277-283.

Dallman MA, Ladle DR (2013) Quantitative analysis of locomotor defects in neonatal mice lacking proprioceptive feedback. Physiol Behav 120:97105.

Dattani M, Preece M (2004) Growth hormone deficiency and related disorders: insights into causation, diagnosis, and treatment. Lancet 363:19771987.

De Lima J, Alvares D, Hatch DJ, Fitzgerald M (1999) Sensory hyperinnervation after neonatal skin wounding: effect of bupivacaine sciatic nerve block. Br J Anaesth 83:662-664.

Dehkhoda F, Lee CMM, Medina J, Brooks AJ (2018) The growth hormone receptor: mechanism of receptor activation, cell signaling, and physiological aspects. Front Endocrinol (Lausanne) 9:35.

Dubick MN, Ravin TH, Michel Y, Morrisette DC (2015) Use of localized human growth hormone and testosterone injections in addition to manual therapy and exercise for lower back pain: a case series with 12-month follow-up. J Pain Res 8:295-302.

Farris GM, Miller GK, Wollenberg GK, Molon-Noblot S, Chan C, Prahalada S (2007) Recombinant rat and mouse growth hormones: risk assessment of carcinogenic potential in 2-year bioassays in rats and mice. Toxicol Sci 97:548-561.

Feather-Schussler DN, Ferguson TS (2016) A battery of motor tests in a neonatal mouse model of cerebral palsy. J Vis Exp. Advance online publication. Retrieved Nov 3, 2016. doi: 10.3791/53569.

Fitzgerald M (2005) The development of nociceptive circuits. Nat Rev Neurosci 6:507-520.

Ford ZK, Dourson AJ, Liu X, Lu P, Green KJ, Hudgins RC, Jankowski MP (2019) Systemic growth hormone deficiency causes mechanical and thermal hypersensitivity during early postnatal development. IBRO Rep 6:111-121.

Gomez R, Kohler DM, Brackley AD, Henry MA, Jeske NA (2018) Serum response factor mediates nociceptor inflammatory pain plasticity. Pain Rep 3:e658.

Goodrich CA (1977) Measurement of body temperature in neonatal mice. J Appl Physiol Respir Environ Exerc Physiol 43:1102-1105.

Gouin O, L'Herondelle K, Lebonvallet N, Le Gall-Ianotto C, Sakka M, Buhé V, Plée-Gautier E, Carré J-L, Lefeuvre L, Misery L, Le Garrec R (2017) TRPV1 and TRPA1 in cutaneous neurogenic and chronic inflammation: pro-inflammatory response induced by their activation and their sensitization. Protein Cell 8:644-661.

Govers R, ten Broeke T, van Kerkhof P, Schwartz AL, Strous GJ (1999) Identification of a novel ubiquitin conjugation motif, required for ligandinduced internalization of the growth hormone receptor. EMBO J 18:2836.

Hermann C, Hohmeister J, Demirakça S, Zohsel K, Flor H (2006) Long-term alteration of pain sensitivity in school-aged children with early pain experiences. Pain 125:278-285.

Hester MS, Danzer SC (2013) Accumulation of abnormal adult-generated hippocampal granule cells predicts seizure frequency and severity. J Neurosci 33:8926-8936.

Jankowski MP, McIlwrath SL, Jing X, Cornuet PK, Salerno KM, Koerber HR, Albers KM (2009) Sox11 transcription factor modulates peripheral nerve regeneration in adult mice. Brain Res 1256:43-54.

Jankowski MP, Ross JL, Weber JD, Lee FB, Shank AT, Hudgins RC (2014) Age-dependent sensitization of cutaneous nociceptors during developmental inflammation. Mol Pain 10:34.

Julius D, Basbaum AI (2001) Molecular mechanisms of nociception. Nature 413:203-210.

Kao DJ, Li AH, Chen JC, Luo RS, Chen YL, Lu JC, Wang HL (2012) CC chemokine ligand 2 upregulates the current density and expression of
TRPV1 channels and Nav1.8 sodium channels in dorsal root ganglion neurons. J Neuroinflammation 9:189.

Koerber HR, Woodbury CJ (2002) Comprehensive phenotyping of sensory neurons using an ex vivo somatosensory system. Physiol Behav 77:589594.

Koerber HR, McIlwrath SL, Lawson JJ, Malin SA, Anderson CE, Jankowski MP, Davis BM (2010) Cutaneous C-polymodal fibers lacking TRPV1 are sensitized to heat following inflammation, but fail to drive heat hyperalgesia in the absence of TPV1 containing C-heat fibers. Mol Pain 6:58.

Kumar SK, Bhat BV (2016) Distinct mechanisms of the newborn innate immunity. Immunol Lett 173:42-54.

Kumar PA, Chitra PS, Lu C, Sobhanaditya J, Menon R (2014) Growth hormone $(\mathrm{GH})$ differentially regulates NF-kB activity in preadipocytes and macrophages: implications for GH's role in adipose tissue homeostasis in obesity. J Physiol Biochem 70:433-440.

Lau J, Minett MS, Zhao J, Dennehy U, Wang F, Wood JN, Bogdanov YD (2011) Temporal control of gene deletion in sensory ganglia using a tamoxifen-inducible Advillin-Cre-ERT2 recombinase mouse. Mol Pain 7:100.

Leone S, Chiavaroli A, Recinella L, Orlando G, Ferrante C, Marconi GD, Gasparo I, Bitto A, Salvatori R, Brunetti L (2019) Increased pain and inflammatory sensitivity in growth hormone-releasing hormone (GHRH) knockout mice. Prostaglandins Other Lipid Mediat 144:106362.

Lim Y, Godambe S (2017) Prevention and management of procedural pain in the neonate: an update, American Academy of Pediatrics, 2016. Arch Dis Child Educ Pract Ed 102:254-256.

Liu X, Green KJ, Ford ZK, Queme LF, Lu P, Ross JL, Lee FB, Shank AT, Hudgins RC, Jankowski MP (2017) Growth hormone regulates the sensitization of developing peripheral nociceptors during cutaneous inflammation. Pain 158:333-346.

Lu C, Kumar PA, Sun J, Aggarwal A, Fan Y, Sperling MA, Lumeng CN, Menon RK (2013) Targeted deletion of growth hormone (GH) receptor in macrophage reveals novel osteopontin-mediated effects of GH on glucose homeostasis and insulin sensitivity in diet-induced obesity. J Biol Chem 288:15725-15735.

Marsh D, Dickenson A, Hatch D, Fitzgerald M (1999) Epidural opioid analgesia in infant rats II: responses to carrageenan and capsaicin. Pain 82:33-38.

McGrath-Morrow SA, Lee S, Gibbs K, Lopez A, Collaco JM, Neptune E, Soloski MJ, Scott A, D’Alessio F (2015) Immune response to intrapharyngeal LPS in neonatal and juvenile mice. Am J Respir Cell Mol Biol 52:323-331.

McKelvey R, Berta T, Old E, Ji RR, Fitzgerald M (2015) Neuropathic pain is constitutively suppressed in early life by anti-inflammatory neuroimmune regulation. J Neurosci 35:457-466.

McMahon SB, Cafferty WB, Marchand F (2005) Immune and glial cell factors as pain mediators and modulators. Exp Neurol 192:444-462.

McMahon SB, La Russa F, Bennett DL (2015) Crosstalk between the nociceptive and immune systems in host defence and disease. Nat Rev Neurosci $16: 389-402$.

Mendell LM (1996) Neurotrophins and sensory neurons: role in development, maintenance and injury. A thematic summary. Philos Trans R Soc Lond B Biol Sci 351:463-467.

Moqrich A, Earley TJ, Watson J, Andahazy M, Backus C, Martin-Zanca D, Wright DE, Reichardt LF, Patapoutian A (2004) Expressing TrkC from the TrkA locus causes a subset of dorsal root ganglia neurons to switch fate. Nat Neurosci 7:812-818.

Moriarty O, Harrington L, Beggs S, Walker SM (2018) Opioid analgesia and the somatosensory memory of neonatal surgical injury in the adult rat. $\mathrm{Br}$ J Anaesth 121:314-324.

Moriarty O, Tu Y, Sengar AS, Salter MW, Beggs S, Walker SM (2019) Priming of adult incision response by early-life injury: neonatal microglial inhibition has persistent but sexually dimorphic effects in adult rats. J Neurosci 39:3081-3093.

Müller EE, Locatelli V, Cocchi D (1999) Neuroendocrine control of growth hormone secretion. Physiol Rev 79:511-607.

Nikolaou S, Hu L, Cornwall R (2015) Afferent innervation, muscle spindles, and contractures following neonatal brachial plexus injury in a mouse model. J Hand Surg Am 40:2007-2016.

Philippou A, Maridaki M, Theos A, Koutsilieris M (2012) Cytokines in muscle damage. Adv Clin Chem 58:49-87. 
Pinho-Ribeiro FA, Baddal B, Haarsma R, O’Seaghdha M, Yang NJ, Blake KJ, Portley M, Verri WA, Dale JB, Wessels MR, Chiu IM (2018) Blocking neuronal signaling to immune cells treats streptococcal invasive infection. Cell 173:1083-1097.e22.

Queme LF, Ross JL, Lu P, Hudgins RC, Jankowski MP (2016) Dual modulation of nociception and cardiovascular reflexes during peripheral ischemia through $\mathrm{P} 2 \mathrm{Y} 1$ receptor-dependent sensitization of muscle afferents. J Neurosci 36:19-30.

Queme LF, Weyler AA, Cohen ER, Hudgins RC, Jankowski MP (2020) A dual role for peripheral GDNF signaling in nociception and cardiovascular reflexes in the mouse. Proc Natl Acad Sci USA 117:698-707.

Ren K, Dubner R (2010) Interactions between the immune and nervous systems in pain. Nat Med 16:1267-1276.

Ren K, Anseloni V, Zou SP, Wade BE, Novikova IS, Ennis M, Traub JR, Gold SM, Dubner R, Lidow SM (2004) Characterization of basal and re-inflammation-associated long-term alteration in pain responsivity following short-lasting neonatal local inflammatory insult. Pain 110:588-596.

Ross JL, Queme LF, Cohen ER, Green KJ, Lu P, Shank AT, An S, Hudgins RC, Jankowski MP (2016) Muscle IL1 $\beta$ drives ischemic myalgia via ASIC3-mediated sensory neuron sensitization. J Neurosci 36:6857-6871.

Ross JL, Queme LF, Lamb JE, Green KJ, Jankowski MP (2018) Sex differences in primary muscle afferent sensitization following ischemia and reperfusion injury. Biol Sex Differ 9:2.

Salaffi F, Giacobazzi G, Di Carlo M (2018) Chronic pain in inflammatory arthritis: mechanisms, metrology, and emerging targets-a focus on the JAK-STAT pathway. Pain Res Manag 2018:8564215.

Sass F, Fuchs M, Pumberger M, Geissler S, Duda G, Perka C, Schmidt-Bleek $\mathrm{K}$ (2018) Immunology guides skeletal muscle regeneration. Int J Mol Sci 19:835.

Schindelin J, Arganda-Carreras I, Frise E, Kaynig V, Longair M, Pietzsch T, Preibisch S, Rueden C, Saalfeld S, Schmid B, Tinevez JY, White DJ, Hartenstein V, Eliceiri K, Tomancak P, Cardona A (2012) Fiji: an opensource platform for biological-image analysis. Nat Methods 9:676-682.

Schneider A, Wood HN, Geden S, Greene CJ, Yates RM, Masternak MM, Rohde KH (2019) Growth hormone-mediated reprogramming of macrophage transcriptome and effector functions. Sci Rep 9:19348.

Schwaller F, Beggs S, Walker SM (2015) Targeting p38 mitogen-activated protein kinase to reduce the impact of neonatal microglial priming on incision-induced hyperalgesia in the adult rat. Anesthesiology 122:13771390.

Sharma N, Flaherty K, Lezgiyeva K, Wagner DE, Klein AM, Ginty DD (2020) The emergence of transcriptional identity in somatosensory neurons. Nature 577:392-398.
Shelton DL, Reichardt LF (1984) Expression of the beta-nerve growth factor gene correlates with the density of sympathetic innervation in effector organs. Proc Natl Acad Sci USA 81:7951-7955.

Sommer C, Kress M (2004) Recent findings on how proinflammatory cytokines cause pain: peripheral mechanisms in inflammatory and neuropathic hyperalgesia. Neurosci Lett 361:184-187.

Strous GJ, van Kerkhof P, Govers R, Ciechanover A, Schwartz AL (1996) The ubiquitin conjugation system is required for ligand-induced endocytosis and degradation of the growth hormone receptor. EMBO J 15:38063812.

van der Lely AJ, Hutson RK, Trainer PJ, Besser GM, Barkan AL, Katznelson L, Klibanski A, Herman-Bonert V, Melmed S, Vance ML, Freda PU, Stewart PM, Friend KE, Clemmons DR, Johannsson G, Stavrou S, Cook DM, Phillips LS, Strasburger CJ, Hacker S, et al. (2001) Long-term treatment of acromegaly with pegvisomant, a growth hormone receptor antagonist. Lancet 358:1754-1759.

Walker SM (2019) Long-term effects of neonatal pain. Semin Fetal Neonat Med 24:101005.

Walker SM, Tochiki KK, Fitzgerald M (2009) Hindpaw incision in early life increases the hyperalgesic response to repeat surgical injury: critical period and dependence on initial afferent activity. Pain 147:99-106.

Walker SM, Beggs S, Baccei ML (2016) Persistent changes in peripheral and spinal nociceptive processing after early tissue injury. Exp Neurol 275:253-260.

Walker SM, O’Reilly H, Beckmann J, Marlow N (2018) Conditioned pain modulation identifies altered sensitivity in extremely preterm young adult males and female. Br J Anaesth 121:636-646.

Weston WL, Carson BS, Barkin RM, Slater GD, Dustin RD, Hecht SK (1977) Monocyte-macrophage function in the newborn. Am J Dis Child 131:1241-1242.

Winterberg T, Vieten G, Meier T, Yu Y, Busse M, Hennig C, Hansen G, Jacobs R, Ure BM, Kuebler JF (2015) Distinct phenotypic features of neonatal murine macrophages. Eur J Immunol 45:214-224.

Ye Y, Woodbury CJ (2010) Early postnatal loss of heat sensitivity among cutaneous myelinated nociceptors in Swiss-Webster mice. J Neurophysiol 103:1385-1396.

Zhang X, Azhar G, Rogers SC, Foster SR, Luo S, Wei JY (2014) Overexpression of p49/STRAP alters cellular cytoskeletal structure and gross anatomy in mice. BMC Cell Biol 15:32-32.

Zhong XS, Winston JH, Luo X, Kline KT, Nayeem SZ, Cong Y, Savidge TC, Dashwood RH, Powell DW, Li Q (2018) Neonatal colonic inflammation epigenetically aggravates epithelial inflammatory responses to injury in adult life. Cell Mol Gastroenterol Hepatol 6:65-78. 